\title{
WestVirginiaUniversity
}

THE RESEARCH REPOSITORY @ WVU

Graduate Theses, Dissertations, and Problem Reports

1999

\section{Effects of gender and social support on cardiovascular reactivity to a speech task}

Lynda M. Ciano-Federoff

West Virginia University

Follow this and additional works at: https://researchrepository.wvu.edu/etd

\section{Recommended Citation}

Ciano-Federoff, Lynda M., "Effects of gender and social support on cardiovascular reactivity to a speech task" (1999). Graduate Theses, Dissertations, and Problem Reports. 3161.

https://researchrepository.wvu.edu/etd/3161

This Dissertation is protected by copyright and/or related rights. It has been brought to you by the The Research Repository @ WVU with permission from the rights-holder(s). You are free to use this Dissertation in any way that is permitted by the copyright and related rights legislation that applies to your use. For other uses you must obtain permission from the rights-holder(s) directly, unless additional rights are indicated by a Creative Commons license in the record and/ or on the work itself. This Dissertation has been accepted for inclusion in WVU Graduate Theses, Dissertations, and Problem Reports collection by an authorized administrator of The Research Repository @ WVU.

For more information, please contact researchrepository@mail.wvu.edu. 
Effects of Gender and Social Support on Cardiovascular Reactivity to a Speech Task

Lynda M. Ciano-Federoff

\author{
Dissertation submitted to the \\ College of Arts and Sciences \\ at West Virginia University \\ in partial fulfillment of the requirements \\ for the degree of \\ Doctor of Philosophy \\ in \\ Clinical Psychology
}

Kevin T. Larkin, Ph.D., Chair

Jeannie S. Clark, Ph.D.

Stan Cohen, Ph.D.

Barry Edelstein, Ph.D.

William Fremouw, Ph.D.

Department of Psychology

Morgantown, West Virginia

1999

Keywords: Cardiovascular Reactivity, Social Support

Copyright 1999, Lynda M. Ciano-Federoff 


\begin{abstract}
Effects of Gender and Social Support on Cardiovascular Reactivity to a Speech Task
\end{abstract}

\title{
Lynda M. Ciano-Federoff
}

The effects of gender and social support on cardiovascular reactivity to a speech task were examined in this study. Seventeen males and seventeen females performed a speech task, once in the presence of an experimenter with their significant other present offering encouragement and support and once in the presence of the experimenter alone. Both blood pressure (i.e., systolic (SBP) and diastolic (DBP)) and hemodynamic parameters (i.e., heart rate (HR), cardiac output (converted to cardiac index, CI), stroke volume (converted to stroke index, SI), and pre-ejection period (PEP)) were measured during the speech tasks. In addition, total peripheral resistance (TPR) was calculated to examine the effects of the social support manipulation on the vasculature. Performance of the speech tasks was associated with increased SBP, DBP, and HR responses, as well as increased CI and TPR and decreased PEP. Females exhibited higher HR, SI, and CI and lower PEP and TPR than males during the speech tasks. Males exhibited a greater reduction in SI during both preparatory and speech phases than females. No main or interaction effects for social support were noted. These findings suggest that the presence of a significant other may not be sufficient to attenuate cardiovascular reactivity to a speech task and that other more subtle factors may be important.

This dissertation was funded by grants from the West Virginia University Doctoral Student Research Program and the Department of Psychology Alumni Fund for Graduate and Undergraduate Student Research and Travel. 


\section{Author's Acknowledgments}

The author wishes to acknowledge individuals who helped with the formulation of this project including Kevin Larkin, Dan Hollander, and Jeffrey Goodie, as well as individuals who assisted with data collection including Dan Hollander, Bill Federoff, and Emily Dailey. Special thanks also goes out to my committee members for their efforts in shaping this document, especially Kevin Larkin for his numerous hours spent editing this work. And last, but certainly not least, thanks to my husband Bill and our daughter Tara for their patience and love during the hours I spent completing this dissertation. 


\section{Table of Contents}

Introduction

Cardiovascular Reactivity to Stress and Health $\quad 2$

Social Support and Cardiovascular Reactivity to Stress 3

Influential Factors in Cardiovascular Research 12

$\begin{array}{ll}\text { Statement of Purpose } & 13\end{array}$

$\begin{array}{ll}\text { Method } & 18\end{array}$

$\begin{array}{lr}\text { Participants } & 18\end{array}$

$\begin{array}{ll}\text { Design } & 19\end{array}$

$\begin{array}{ll}\text { Apparatus } & 19\end{array}$

$\begin{array}{ll}\text { Experimental task } & 20\end{array}$

$\begin{array}{ll}\text { Self-report Questionnaires } & 21\end{array}$

$\begin{array}{ll}\text { Procedures } & 23\end{array}$

$\begin{array}{ll}\text { Results } & 26\end{array}$

$\begin{array}{ll}\text { Discussion } & 34\end{array}$

$\begin{array}{ll}\text { References } & 53\end{array}$

$\begin{array}{ll}\text { Tables } & 60\end{array}$

$\begin{array}{ll}\text { Figure Captions } & 66\end{array}$

$\begin{array}{ll}\text { Figures } & 67\end{array}$

$\begin{array}{ll}\text { Appendices } & 72\end{array}$

$\begin{array}{ll}\text { Curriculum Vita } & 76\end{array}$ 


\section{List of Multimedia Objects}

Table 1. Descriptive Statistics for Demographic Variables by Gender $(n=34)$. Page 60.

Table 2. Descriptive Statistics (Standard Deviations in parentheses) for all cardiovascular parameters chronologically during Rest, Preparation, and Speech phases $(\mathrm{n}=34)$. Page 61 .

Table 3. Descriptive statistics for Stress and Arousal Subscales of the Stress/Arousal Checklist (chronologically and by Condition). Page 63.

Table 4. Partial correlation coefficients for MSIS and FNE scores with cardiovascular difference scores by Condition. Page 64.

Figure 1. DBP (mm Hg) by Order (Partner Present First, No Partner Present First) and Period (i.e., first speech, second speech). Page 67.

Figure 2. Adjusted Mean HR (bpm) by Gender and Phase (preparation, speech). Page 68.

Figure 3. Adjusted Mean PEP (ms) by Gender and Phase (preparation, speech). Page 69.

Figure 4. Cardiac Index (\% change) by Gender and Phase (preparation, speech). Page 70.

Figure 5. TPR (\% change) by Gender and Phase (preparation, speech). Page 71. 
Effects of Gender and Social Support on Cardiovascular Reactivity to a Speech Task

If modern medicine is to begin integrating disease prevention strategies with traditional medical treatment of disease in the twenty-first century and beyond, it is imperative that factors that promote or preserve health be identified and exploited. Although medical research has identified many factors that cause or promote illness (e.g., carcinogens, stress), few factors have been identified that enhance or promote health. In addition, those empirically identified and verified relations between environmental risk factors and health (both physiological and psychological) that have been observed have been based largely on correlational research. Studies that encourage more causal attributions between specific healthy behaviors and overall indicators of health remain in their infancy.

One promising factor that has been investigated using varied methodologies (e.g., correlational, experimental, prospective studies) and has been found to be consistently related to measures of overall health is social support. For example, epidemiological studies (e.g., Berkman \& Syme, 1979; Carter \& Glick, 1970) have revealed that all-cause mortality was lower among married people than among people who were not married. Also, individuals who were relatively socially isolated, independent of age or initial health status, suffered poorer health than more socially integrated individuals (House, Landis, \& Umberson, 1988; Leppin \& Schwarzer, 1990). The term "social support" assumes, for the purpose of this study, that the individual exists within a social context (i.e., rather than being socially isolated) and that this social context provides opportunities for interaction that the individual finds valuable or pleasurable in some way.

Despite the empirical work supporting the salubrious effects of both social interactions and the resulting support obtained from these interactions upon overall health, very little is 
known regarding the physiological mechanisms involved in explaining how social phenomena impact biopsychological functioning. One hypothesis that has received some attention in explaining this socialbiological relation focuses upon the ability of social support to facilitate more adaptive physiological responses to environmental stress (Cassell, 1976). It has been firmly established that characteristic cardiovascular, adrenocorticotrophic, and immunological alterations occur when an organism is exposed to both acute and chronic forms of environmental stress (Uchino, Cacioppo, \& KiecoltGlaser, 1996). Borrowing a term from Selye's (1956) original work, physiological systems of socially supported persons might experience less "strain" than those of less supported persons, resulting in better overall health. According to this hypothesis, then, the magnitude of these physiological responses to stress is attenuated (and, thus, subjected to less strain) for persons well integrated into a social network in contrast to persons experiencing very little social support or social integration. Because the beneficial aspects of social relationships have been widely observed to impact a number of medical conditions including coronary heart disease, infectious illnesses, and cancer, as well as cardiovascular disease (Uchino et al., 1996), the leading cause of death in industrialized nations, examination of the physiological mechanisms responsible for the beneficial attributes of social support is clearly warranted.

Cardiovascular Reactivity to Stress and Health

One variable that has been proposed to quantify the amount of strain endured by the cardiovascular system and that has demonstrated an attenuating effect as a result of social support is cardiovascular reactivity to mental stress. The term "cardiovascular reactivity" refers to the nature and magnitude of an individual's cardiovascular response (e.g., blood pressure, heart rate) to environmental and laboratory demands or threats (Krantz and Manuck, 1984). Stressful tasks 
employed in these studies include both mental (e.g., cognitive challenges and stressful interviews) and physical challenges (i.e., static or dynamic exercise tasks). The "reactivity hypothesis" postulates that an individual's exaggerated cardiovascular reactivity to such stressors could lead to cardiovascular disease over time (Clarkson, Manuck, \& Kaplan, 1986). That is, individuals who react to stress with an exaggerated cardiovascular response to stress (i.e., heart rate, blood pressure) are at increased risk of developing hypertension and/or coronary artery disease later in life (Krantz \& Manuck, 1984). Although there is considerable empirical support for this hypothesis in both animal (e.g., Clarkson et al., 1986; Folkow, Hallback. Lundgren, Sivertsson, \& Weiss, 1973; Manuck, Kaplan, \& Clarkson, 1983) and human models (e.g., Keys et al., 1971), some research has failed to support the reactivity hypothesis (Rosenman, 1990).

Social Support and Cardiovascular Reactivity to Stress

Researchers have examined the relation between social support and cardiovascular parameters, both basal (resting) and phasic measures (reactive), using different methodologies. For example, Cottington, Brock, House, and Hawthorne (1985) examined the relation between perceived quality of life and basal systolic blood pressure (SBP) and diastolic blood pressure (DBP) in normotensive females and males. They found that females who reported poorer social support had marginally higher resting SBPs than the other groups (i.e., all males and females who reported higher levels of social support). Janes (1990) found that persons with more social resources (e.g., church attendance, close friends in the area) and higher instrumental support (e.g., help from friends and relatives with child care or job searches) had lower basal SBP and DBP than persons with fewer social resources or lower instrumental support. Bland, Krough, Winkelstein, and Trevisan (1991) found that a larger social 
network (e.g., number of persons in household, number of siblings, frequency of religious service attendance, etc.) was correlated with lower basal SBP and DBP for both females and males. Correlational studies have supported the hypothesis that degree of social support is positively associated with cardiovascular functioning at rest (Uchino et al., 1996). Of course, the nature of correlational studies limits the causal inferences that may be made as a result of this work.

Another type of study that has been used to examine the relation between social support and cardiovascular parameters is the intervention study. For example, Andersson (1985) assessed resting SBP and DBP in self-reported lonely, elderly women. The researchers provided social support during the intervention portion of the study in the form of small discussion groups. They found that the group of females who completed the group discussion procedure had lower basal SBP and DBP after the intervention than the control group of females who did not participate in the group discussions. Again, in general, the intervention studies reviewed by Uchino et al. (1996) supported the hypothesis that improved social support/enhanced social network positively impacts the cardiovascular system by reducing basal SBP and DBP.

In addition, several situationally-focused empirical studies have manipulated social support (e.g., by manipulating social situations) as the independent variable and examined the effect on cardiovascular reactivity (i.e., blood pressure and, in most cases, heart rate (HR)) as the dependent variables. In contrast to previous works that have examined the effect of social support upon resting cardiovascular parameters, these studies have measured cardiovascular reactions to mentally challenging tasks (Allen, Blascovich, Tomaka, \& Kelsey, 1991; Christenfeld et al., 1997; Edens, Larkin, \& Abel, 1992; Gerin, Milner, Chawla, \& Pickering, 1995; Gerin, Pieper, 
Levy, \& Pickering, 1992; Kamarck, Annunziato, \& Amateau, 1995; Kamarck, Manuck, \& Jennings, 1990; Kleinke \& Williams, 1993; Lepore, 1995; Lepore, Allen, \& Evans, 1993; Sheffield \& Carroll, 1994; Uchino \& Garvey, 1997). Because this paper focuses upon the effect of manipulation of social support conditions upon measures of cardiovascular reactivity, these studies will be reviewed in depth. In 1990, Kamarck et al. were the first researchers to experimentally examine the effects of social support on cardiovascular reactivity to mental tasks. Female participants were asked to choose a female friend to bring to the laboratory with them if requested by the experimenter. Subjects were subsequently randomly assigned to either the group that would perform their tasks alone or the group that would perform their tasks in the presence of the chosen friend. In the Friend condition, the participant was told that the friend was there to give social support. The friend (who was required to wear headphones broadcasting white noise and be engaged in tasks of her own in an attempt to remove any evaluative potential) lightly touched the subject on the wrist during the tasks in order to communicate support. The results of this study showed that those subjects who performed the task in the presence of their friend demonstrated less $\mathrm{HR}$ and SBP reactivity to a mental arithmetic task than subjects who performed the task alone. With regard to a concept formation task, subjects accompanied by their friend displayed less of a HR response than individuals who performed the task alone.

Allen et al. (1991) concentrated on the "evaluative" component of social support that was so well-controlled by Kamarck et al. (1990) and examined how potentially evaluative and nonevaluative others could buffer cardiovascular reactivity to a mental task. In addition, they compared cardiovascular reactivity to laboratory tasks versus tasks performed in a field setting. 
They administered a serial subtraction task to 45 female pet owners in a laboratory and again 2 weeks later at the participant's home in one of three randomly assigned conditions: (a) in the presence of their pet dog, (b) in the presence of a female friend, or (c) alone. All trials both in the laboratory and at the subject's home were performed in the presence of a female experimenter seated unobtrusively behind the participant. No differences were observed in the baseline cardiovascular functioning between participants tested in the three conditions. Results in the home, however, revealed that participants in the Friend condition had significantly higher HR, SBP, and skin conductance reactivity to the mental arithmetic task than participants in the Alone group. The Alone group, in turn, displayed higher reactivity than those subjects who performed the task in the Pet condition. This effect was evident on all physiological parameters measured, including HR, SBP, DBP, and skin conductance. Accuracy of performance was also assessed to evaluate whether the increased arousal of participants in the Friend condition was a result of increased effort on the task. Surprisingly, participants in the Friend condition performed significantly poorer than participants in the other two conditions, although they performed the task in significantly less time than participants in the Pet or Alone conditions. The results of this study are contrary to the pattern of results obtained by Kamarck et al. (1990). The authors explained this discrepancy in results as a consequence of both the evaluative component present in this experiment (i.e., friends were not distracted from observing) and, perhaps, by the lack of physical contact (friends in the Kamarck et al. study touched subjects on the wrist). In addition, the larger attenuating effect of the pets suggested that the lack of an evaluative component (i.e., non-judgmental support of their pet) was an important factor in the attenuating effect.

In another partial replication and extension of the work of Kamarck et al. (1990), Edens et 
al. (1992) observed female subjects performing a mental arithmetic task and a mirror tracer challenge (i.e., subjects traced an outline of a star using only a mirror as a guide) in one of five randomly assigned social conditions: in the presence of a friend of their own choosing, either with the friend (a) touching them lightly on the nondominant arm during the task or (b) merely present; with an observer of the experimenter's choosing present either (c) touching the subject lightly on the nondominant arm, or (d) merely present; or (e) alone. Observers were distracted in a manner similar to the Kamarck et al. (1990) study to remove the evaluative component of the social interaction. They found that subjects in the two touch conditions displayed higher HR, SBP, and DBP reactivity to the mental arithmetic task than subjects in nontouch conditions. They also observed that subjects in the Stranger conditions displayed higher HR and DBP reactivity to the mental arithmetic task than participants who performed the task in the presence of a friend. Subjects who performed the mental arithmetic task alone also displayed higher SBP reactivity than subjects who had their friend merely observe (i.e., no touch). No significant effects were observed for the mirror tracer challenge. No differences in accuracy of performance were observed with regard to either task, nor were there differences on perceived level of social support among the various conditions.

Also in 1992, Gerin et al. examined the effects of social support or lack of social support in a group discussion where the female subject discussed controversial issues. Each subject was part of a group consisting of herself and three of the experimenter's confederates. Two of the confederates in each group argued with the subject about a controversial issue on which the subject had demonstrated extreme views (i.e., either abortion, euthanasia, the death penalty, or gun control). Subjects were randomly assigned to either a group in which the third confederate defended the subject (i.e., was 
supportive) or the other confederate remained quiet (i.e., was not supportive). Subjects in the Support condition experienced significantly lower HR, SBP, and DBP elevations than subjects in the No Support condition.

Kleinke and Williams (1993) examined cardiovascular reactivity to a speech task. They manipulated gender of the experimenter, interviewer status, and whether or not the participant was touched during the task. Interviewer status was manipulated by having either an equal-status experimenter (i.e., dressed casually and introduced as a college sophomore) or a higher status interviewer (i.e., dressed in a suit or dress and introduced as a graduate student knowledgeable about physiology) conduct the experiment. Touch was manipulated by having half the experimenters touch the participant for 2 seconds on the elbow of the nondominant arm immediately before beginning the experiment. Neither gender nor touch effects were observed regarding cardiovascular reactivity. Participants who were interviewed by a higher status researcher displayed higher HRs and SBPs than subjects interviewed by confederates introduced as college sophomores.

In 1993, Lepore et al. assessed the effects of social support and nonsupport. They tested subjects in one of three conditions: (a) subjects were either given support in the form of encouragement and empathetic gestures from a sympathetic confederate during a speech task, (b) subjects were given no support by a confederate during the challenge, or (C) subjects performed the task alone. Results revealed reduced SBP reactivity to the task among participants in the Support condition compared with individuals who performed the speech either in the No Support or Alone conditions. Further, subjects in the Alone condition displayed significantly lower SBP and DBP reactivity than those individuals in the No Support condition. Regarding DBP, participants who performed the task with Support displayed 
supportive) or the other confederate remained quiet (i.e., was not participants who performed the task in the No Support condition only. There was no difference between subjects in the Support and Alone condition on DBP reactivity. In addition, males displayed higher SBP and DBP reactivity to the speech task than females.

Sheffield and Carroll (1994) examined the effect of social condition on cardiovascular reactivity to a mental arithmetic task and a vocabulary task. All subjects were initially tested alone and subsequently tested in either Friend present or Stranger present conditions, employing a repeatedmeasures, within-subjects design. Results of the study did not support the hypothesis that social support attenuated cardiovascular reactivity to laboratory tasks. Subjects tested alone, with a friend, or with a stranger all exhibited a comparable magnitude of cardiovascular reactivity to the tasks in this study.

Gerin et al. (1995) attempted to identify the mechanism by which social support may impact cardiovascular reactivity by manipulating both stress level (i.e., low, high) and social support (i.e., alone, with a roommate) in a within-subjects design. All subjects played the same game on the computer that required them to track moving animals on the computer screen and click on them to prevent them from "escaping" a game preserve. Females in the High Stress condition, however, were verbally urged to go faster and harassed by the experimenter during the task. Females in the Low Stress condition were allowed to complete the task without harassment. Results revealed an interaction between the social support condition and stress condition. Subjects in the High Stress condition exhibited lower cardiovascular reactivity to the computer task in the Support condition than those same subjects in the Alone condition on SBP and DBP (HR was marginally significant). Based on their results, the authors concluded that social support attenuated cardiovascular reactivity under conditions of high, but not low, 
stress.

Kamarck et al. (1995) examined the effects of affiliation (i.e., an Alone condition versus accompanied by a close Friend condition) and social threat (through manipulating social behavior and perceived social status of the experimenter) on cardiovascular reactivity to a mental arithmetic task and the Stroop word-color task. As in their previous study, participants were asked to choose a female friend to bring to the laboratory with them if requested by the experimenter. Subjects were then randomly assigned either to the group that would perform their tasks alone or to the group that would perform their tasks in the presence of the chosen friend. Instructions given to the friends were the same as in their earlier study (i.e., Kamarck et al., 1990). In addition, there was a "social threat" manipulation in this study. In the High-Threat condition, the experimenter introduced himself as a doctor, was dressed formally, wore a lab coat, and interacted with the subject in a very formal, impatient, and impersonal way. In contrast, in the Low-Threat condition, the experimenter introduced himself by his first name, wore casual clothes, and gave instructions in a steady, modulated tone of voice. Explicitly evaluative aspects of the interaction were minimized. Results showed that subjects in the Friend condition displayed lower SBP and DBP reactivity to the tasks in the High-Threat but not the LowThreat condition.

Lepore (1995) studied whether individuals categorized according to level of cynicism manifested lower cardiovascular reactivity to a speech task with a social support manipulation. Lepore required subjects to develop a speech on euthanasia with 2-minutes preparation and then give the speech in front of a video camera and an observation mirror, from which they were told they were being observed. Half of the participants were alone in the room during the task and half were observed 
by a supportive observer who was carefully trained to be equally supportive to all subjects. Results showed that those subjects who performed the speech in the presence of a supportive confederate displayed smaller HR, SBP, and DBP reactivity to the speech task than those individuals in the Alone condition who were told that they were being observed through the glass. No significant gender effects were observed for any of the cardiovascular parameters measured, but there was a trend toward higher HR reactivity for females than males and higher DBP reactivity for males than females. Although there was a trend for participants who were classified as low in cynicism to manifest lower SBPs than all other participants, no effects reached statistical significance. Lepore used the Stress/Arousal Adjective Checklist (SAAC; MacKay, Cox, Burrows, \& Lazzerini, 1978) to assess the perceived stress that subjects reported as a result of the speech task. The SAAC comprises two subscales that measure affective (i.e., psychological) and arousal (i.e., physiological) components of perceived stress. Interestingly, Lepore found that the affective subscale scores were correlated with cardiovascular reactivity and appeared to mediate the effects of social support on the observed reduction in cardiovascular reactivity to the speech task. Conversely, the arousal dimension did not appear to be involved in the link between social support and cardiovascular reactivity to the speech stressor.

Uchino and Garvey (1997) examined the effects of manipulating the availability of social support rather than the effects of enacted social support. Participants were randomly assigned to perform a speech task in either a Support Available condition (i.e., as part of the protocol the experimenter offered assistance to the participant if necessary during the task) or in a No Support Available condition (i.e., the experimenter did not offer assistance as part of the protocol). The data from five participants who actually utilized the assistance were eliminated from the statistical analyses in 
order to isolate the effects of social support availability. Participants in the Support Available condition displayed lower SBP and DBP reactivity to the speech task than participants in the No Support Available condition. Uchino and Garvey suggest that having access to social support may be more important in attenuating cardiovascular reactivity to mental tasks than having actually experienced socially supportive interactions.

Finally, in 1997, Christenfeld et al. manipulated the social support status of an observer during performance of a speech task. Participants were randomly assigned to perform a speech task in front of a Stranger or in front of a Supportive Friend. Strangers were either trained to be supportive or neutral during the task. Participants who performed the task in the presence of a supportive observer (both Friend and Supportive Stranger) showed significantly smaller cardiovascular increases than subjects who performed the task in the presence of a Neutral Stranger. Further, the participants in the Friend condition produced smaller SBP responses to the speech task than subjects in the Supportive Stranger condition.

\section{Influential Factors in Cardiovascular Research}

In order to evaluate the methodological "soundness" of these studies and to select those aspects that are important to consider in designing a study examining the effect of social support on cardiovascular reactivity, one finds an effective guide in the work of Rosenman (1990). Rosenman reviewed results from many studies that examined the effects of varying environmental factors on cardiovascular response. He concluded that the following parameters are potentially confounding influences and should be attended to in any reactivity studies. These factors include: age and gender of subject, task type (i.e., active versus passive coping), cognitive task appraisal, body mass, body 
position during testing, response to and duration of abstinence from medication with cardiovascular effects, family and individual health histories including history of hypertension, sleep condition and fatigue level, physical conditioning, failure to account for all cardiovascular/sympathetic nervous system parameters (e.g., HR responses, catecholamine responses, blood volume), and duration of abstinence from alcohol, nicotine, and caffeine. Using these guidelines with the body of literature examining the effect of social support on cardiovascular reactivity to mental stress, it is quite evident that many investigators have failed to account for the influence of many of these potentially confounding variables (e.g., no studies have measured body mass, catecholamine responses, recent sleep history and fatigue level, physical conditioning, or blood volume). Future examinations of how social support impacts cardiovascular reactivity to tasks will need to control more of these factors that have been shown to impact cardiovascular reactivity to tasks (Rosenman, 1990). Despite the methodological problems associated with this body of literature, the consistency of findings is striking. While not all studies have evidenced attenuated cardiovascular reactions to stress among social support conditions (e.g., Sheffield \& Carroll, 1994), the vast majority have observed such an effect (e.g., Christenfeld et al., 1997; Kleinke \& Williams, 1993; Lepore, 1995; Lepore et al., 1993; Uchino \& Garvey, 1997). This beneficial effect appears to exert its greatest influence under highly stressful situations (e.g., Kamarck et al., 1990) and when friends do not exhibit social evaluative behaviors (e.g., Kamarck et al., 1995). The importance of physical touch, however, has been less consistent.

\section{Statement of Purpose}

The purpose of this study is to elaborate upon two features that, at present, have not been examined in this body of literature. The first purpose of this investigation addresses the specific 
cardiovascular mechanisms responsible for the attenuated HR or blood pressure reactivity to a task observed when performed in the presence of a supportive partner as opposed to in the presence of a neutral experimenter. The second purpose of this study is to examine how history of hypertension, sleep condition and fatigue level, physical conditioning, failure to account cardiovascular reactivity to a speech task is differentially affected by social support between males and females.

Regarding the first purpose, it is well known that measures of HR and blood pressure represent only two measurable components of the cardiovascular system and changes in either can reflect many underlying neural or cardiovascular processes. For example, reductions in HR can reflect reduced sympathetic nervous system activity or enhanced parasympathetic nervous system activity. Likewise, reductions in blood pressure could result from reductions in the amount of blood ejected from the heart (i.e., cardiac output $(\mathrm{CO})$ ) or from changes in resistance to the blood flow in the vasculature (i.e., total peripheral resistance (TPR)). Because the only studies to date examining the relation between social support and cardiovascular reactivity to stress have measured only HR and/or blood pressure, very little is known regarding these underlying neural-hemodynamic mechanisms.

Impedance cardiography can be utilized to identify these precise neural-hemodynamic mechanisms that result in blood pressure and HR changes. Impedance cardiography is a noninvasive procedure that can distinguish both mechanisms affecting blood pressure and HR change. Regarding blood pressure changes, this instrument can detect whether alterations in CO or TPR are instrumental in the observed change in blood pressure. Increased CO occurs as the heart pumps a greater amount of blood volume through an unchanged vascular system. Because more blood is going through the same 
vasculature per unit time, arterial pressure in the cardiovascular system increases. Conversely, increased TPR occurs when there is a change in the vasculature (e.g., vasoconstriction). For example, if the heart maintains a relatively constant output of blood but pumps it through a relatively constricted vasculature, the pressure will, again, be increased. Impedance cardiography can reliably differentiate between the relative contributions of these two separate mechanisms (i.e., increased CO or TPR) that are responsible for the change in blood pressure (Miller \& Horvath, 1978). In addition, the impedance cardiograph provides an estimate of a systolic time interval known as pre-ejection period (PEP). Technically the measure from the arrival of the neural signal at the atrial ventricular node on the heart to the opening of the aortic valve (indicating that ejection has begun), PEP represents a measure of \$adrenergic influence on the heart, independent of vagal (i.e., parasympathetic) influence (Cacioppo, Berntson, Binkley, Quigley, Uchino, \& Fieldstone, 1994). As such, the impedance cardiograph can also differentiate HR changes resulting from sympathetic and parasympathetic nervous system activity. Therefore, through use of this instrument, more can be learned about the precise neural-hemodynamic mechanisms that account for the differential reductions in blood pressure or HR observed during social support conditions. Because this study utilizes a within subjects design, we can observe and quantify precisely which mechanisms account for blood pressure and HR changes in each subject performing the task alone versus performing the task in the presence of a supportive partner. Although impedance cardiography has been utilized within a social paradigm previously (e.g., Girdler, Turner, Sherwood, \& Light, 1990), it has not been used during a social support manipulation protocol. Therefore, this proposed study will be the first known study to measure neural-hemodynamic mechanisms responsible for HR and blood pressure reductions that occur under conditions of social support. 
In addition to examining the precise neural-hemodynamic mechanisms of adjustments in cardiovascular reactivity to stress during socially supportive conditions, this study will also test both male and female subjects within the same protocol. Although gender differences in hemodynamic patterning of cardiovascular reactivity to mental stressors have been previously investigated (e.g., Allen, Stoney, Owens, \& Matthews, 1993; Girdler, Turner, Sherwood, \& Light, 1990; Hurwitz, et al., 1993; Lawler, Wilcox, \& Anderson, 1995), results of these studies have been inconclusive. Based on the literature in this area, there is some indication that females are more myocardially reactive than males, and that males are more TPR reactive than females to some tasks (Allen et al., 1993). However, the nature of the tasks utilized in these studies (e.g., reading aloud, speech tasks (without a social support component), math tasks, mirror tracing, Stroop task, handgrip, cold pressor, etc.) have not involved manipulation of social support.

When considering the task chosen for this study, several factors were assessed. First, an active coping task (i.e., a task that requires instrumental coping) was chosen over a passive coping task (e.g., a task in which the participant has no control) because active coping tasks have been shown to increase cardiovascular reactivity more than passive coping tasks (Sherwood, Dolan, \& Light, 1990). Second, when considering the nature of the mental stressor, a speech task was chosen over other mental stressors due to the inherent social characteristics of the task (i.e., communicating to other persons). Also, a speech task has been successfully used in studies that assessed cardiovascular reactivity to tasks involving social support manipulations (e.g., Christenfeld et al., 1997; Kleinke \& Williams, 1993; Lepore, 1995; Lepore et al., 1993; Uchino \& Garvey, 1997). Other mental tasks that have been employed in the social support literature (e.g., mirror tracing, mental arithmetic, vocabulary task, etc.) 
do not have the same inherent social characteristics associated with the task. The last consideration addressed the consistency of results obtained from earlier studies. Studies that have used interpersonal tasks (e.g., Christenfeld et al., 1997; Gerin et al., 1992; Kleinke \& Williams, 1993; Lepore, 1995; Lepore et al., 1993; Uchino \& Garvey, 1997) have resulted in more consistent observations of attenuated cardiovascular reactivity under social support conditions across parameters (including HR, SBP, and DBP) than tasks that are less interpersonal in nature (e.g., mental arithmetic, vocabulary; Sheffield \& Carroll, 1994). Although previous studies have considered the effects of touch on cardiovascular reactivity to mental stressors (e.g., Edens et al., 1992; Kamarck et al, 1990), including a speech task (Kleinke \& Williams, 1993), touch manipulations have not been linked reliably to attenuated cardiovascular responses (e.g., Eden et al., 1992). Further, social support effects on cardiovascular reactivity have been observed in the absence of touch (e.g., Christenfeld et al., 1997; Gerin et al., 1992; Lepore, 1995; Lepore et al., 1993; Uchino \& Garvey, 1997). Therefore, a touch manipulation was not employed in this study.

Addressing the type of social support used in this study, Uchino and Garvey (1997) have examined the hypothesis that there are two dimensions of social support: "available" and "enacted" (Tardy, 1985). Although they found that the "available" dimension was an important factor in the attenuating effect of social support on cardiovascular reactivity to a mental task, their study is the only investigation to examine this parameter. All other investigations have employed social support manipulations that used what Uchino and Garvey would have termed "enacted" social support. Congruent with most of the previous work in this area, the "enacted" dimension was examined in this study. Also, no studies of social support and cardiovascular reactivity have utilized significant others as 
the source of support. If social support attenuates the cardiovascular response to stress as a result of familiarity or comfort with the support person, then the closer the relationship, the greater the attenuating response may be. As a result of this hypothesis, significant others (relationship duration: 2 month's minimum) were utilized as the source of support in this study.

\section{Method}

\section{$\underline{\text { Participants }}$}

A sample size of 16 participants per cell was chosen to achieve a power of 0.80 for " $=.05$, assuming a medium effect size. Therefore, a total of 32 participants was required, including 16 females and 16 males. The final sample size utilized in this study was actually 34 participants, including 17 males and 17 females (see Table 1). Participants were recruited through the West Virginia University (WVU) Department of Psychology subject pool. Persons who were involved in a romantic relationship and who had been involved in that supportive relationship for at least 2 months were actively recruited. Each subject participated in both conditions of the study (i.e., delivery of a speech with their significant other present and without the partner present) on the same day. All participants were screened initially by the experimenter and chosen to participate in the study if they were self-reported healthy individuals who were not on any medication that affected cardiovascular responses, including oral contraceptives. Use of nicotine and family history of hypertension were matched between gender groups (four males and four females smoked, two males and two females had a family history of hypertension; see screening criteria, Appendix A). Participants were asked to refrain from using nicotine products, ingesting caffeine, including chocolate, and food, as well as strenuous exercise for 2 hours before the session. Age, height, weight, race, and number of hours of sleep the night before the experimental session were 
recorded upon arrival at the laboratory (see Appendix B). Upon the completion of the session, the participant was paid $\$ 15.00$ (i.e., approximately $\$ 10 / \mathrm{hr}$ ) and the partner was paid $\$ 5.00$ (i.e., approximately $\$ 10 / \mathrm{hr}$ ).

$\underline{\text { Design }}$

This study employed a 2 X 2 X 2 mixed factors design. The factors were Gender (male, female) X Partner Condition (accompanied by partner, not accompanied by partner) X Phase (speech preparation, speech task) with both Partner Condition and Phase serving as repeated measures. Hemodynamic parameters that were measured included: HR, SBP, DBP, cardiac output (CO), TPR, PEP, and stroke volume (SV).

$\underline{\text { Apparatus }}$

Impedance Measures. An IFM Minnesota Impedance Cardiograph (model \# 304B) was used to measure cardiac PEP, SV, HR, and CO. HR was measured utilizing three chest electrodes that supplied a signal to the IFM Minnesota Cardiograph. Electrodes were placed bilaterally on upper shoulders and lower abdomen of the subject. Results obtained from the impedance cardiograph have been found to be relatively comparable to results obtained from more invasive measures (Miller $\&$ Horvath, 1978; Mohaptra, 1981, as cited in Sherwood \& Turner, 1992). Dry tape aluminized electrodes were attached to each participant and provided information regarding cardiovascular and hemodynamic measures to the impedance cardiograph. The two inner electrodes were voltagedetecting electrodes. One was placed at the base of the neck with the other placed $24 \mathrm{~cm}$ beneath, as recommended by Lamberts, Visser, and Zijlstra (as cited in Kasprowicz, Manuck, Malkoff, \& Krantz, 1990). The two outer electrodes were placed at least $3 \mathrm{~cm}$ away from the nearest voltage-detecting 
electrode. A Hewlett-Packard Contact Sensor (model \#21050A) was placed on each participant's sternum to detect heart sounds. Output from the impedance cardiograph was transmitted directly to an IBM-compatible computer for recording, data reduction, and computation.

Blood Pressure Measures. SBP and DBP measures were obtained every 2 minutes using an IBS SD-700A automated sphygmomanometer, which employs a pressure sensor embedded in an occluding cuff positioned over the brachial artery of the subject's nondominant arm. Inflation/deflation of the cuff was automatically regulated and blood pressure values displayed digitally and recorded by the experimenter.

\section{Experimental task}

One interpersonal speech task, similar to the task used by Christenfeld et al. (1997) and Gerin et al. (1992) was utilized in this experiment. In order to standardize the content of the speeches, each subject was given a fact sheet containing arguments for and against the topics (i.e., euthanasia, abortion, or the death penalty) about which they expressed the most extreme views on prescreening (see Appendix C). One topic was utilized for each Partner Condition (i.e., the participant prepared speeches on different topics for the two social support conditions). Participants were given 4 minutes to prepare a 4-minute speech on each topic. In order to increase each participant's motivation and competitiveness, each participant was told to do his or her best because the speech was recorded, graded, and compared with the speeches of the other participants (Uchino \& Garvey, 1997). The speech was recorded using a JVC video camera to make the stress associated with this procedure more salient, but the tape was reused in each experimental session; no coding was performed. The participant then gave the speech in one of two Partner Conditions: (a) in the presence of a neutral 
experimenter wearing a white laboratory coat, or (b) in the presence of a neutral experimenter wearing a white laboratory coat and their partner. In the Partner-Present condition, the participant was asked to sit across from the partner in the laboratory beside a video camera that recorded the speech. In both Partner Conditions the experimenter stood beside the camera. Following the first speech and the completion of questionnaires and a 6-minute rest phase to allow cardiovascular parameters to return to baseline, each subject was asked to prepare the second speech and perform the task in the alternate condition (i.e., if task was performed first with the partner present, the task was performed subsequently without the partner and vice versa). Partner Conditions were counterbalanced. That is, nine males and eight females were run in the Partner Present condition first. The remaining eight males and nine females were run in the No Partner Present condition first.

\section{$\underline{\text { Self-report questionnaires }}$}

Miller Social Intimacy Scale. The Miller Social Intimacy Scale (MSIS: Miller \& Lefcourt, 1982) was used to assess the stability and quality of the relationship between the participant and her/his partner. The Miller Social Intimacy Scale has 17 items; 6 of which are answered based on a 10-point Likert-type scale ( 1 = "very rarely" to $10=$ "almost always"). An example of one of these questions is: "How often do you feel close to her/him?" The remaining 11 questions are answered based on a 10point Likert-type scale ( $1=$ "not much" to $10=$ "a great deal"). An example of one of these questions is: "How affectionate do you feel toward her/him?" Miller and Lefcourt reported acceptable convergent, discriminant, and construct validity as well as reliability (Cronbach's " = .91; test-retest reliability $\underline{r}=.96$ ) for this relationship measure. Two items (\#2 and \#14) are opposite-keyed and the scores are summed to yield one total score of social intimacy. This instrument was administered to 
determine if a baseline difference in the quality of relationships between gender groups existed.

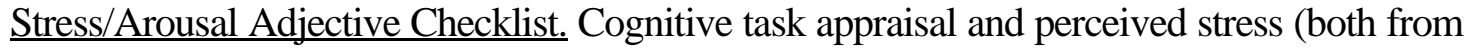
the task and from wearing the impedance electrodes) were assessed using the Stress/Arousal Adjective Checklist (MacKay, Cox, Burrows, \& Lazzerini, 1978). The SAAC consists of 20 adjectives that describe how one feels, both emotionally and physically. Respondents were asked to endorse the degree to which each adjective described their feeling states on a 4-point scale $(0=$ definitely not to 3 $=$ definitely yes). Persons who score high on this measure are reporting higher perceived stress. The 20 items then comprise two 10-item scales (King, Burrows, \& Stanley, 1983); one that measures stress $($ Cronbach's " $=.86)$ and one that measures arousal $($ Cronbach's " $=.74)$.

Fear of Negative Evaluation Questionnaire. The 30-item Fear of Negative Evaluation Questionnaire (FNE; Watson \& Friend, 1969) was utilized to determine the participant's level of social fear associated with evaluative situations. Respondents were asked to answer "true" or "false" to each of the statements as they applied the questions to themselves. The FNE has been found to have acceptable internal consistency (KR-20 $=.94)$, and test-retest reliability $(\mathrm{r}=.78)$. Similarly to the MSIS, because of the social nature of the task, the FNE was utilized to determine if there was a baseline difference between gender groups on their fear of negative evaluation.

Social Support Questionnaire (modified). The Social Support Questionnaire utilized in this study (SSQ6; see Appendix D) was modified from the original SSQ (Sarason, Levine, Basham, \& Sarason, 1983) to reflect the fact that the social support parameter of interest in this study was social support received from the participant's significant other during a specific task. The modified SSQ6 consisted of six questions with a Likert-type ("very dissatisfied" to "very satisfied") answer format. An 
example question from the modified SSQ6 was, "How satisfied were you with the overall support your partner provided to distract you from your worries if you felt under stress?’ This instrument was utilized to assess whether support received from partners during the task (which was standardized across partners) was typical of the level of support received outside the laboratory (i.e., to address ecological validity issues).

$\underline{\text { Procedures }}$

Subjects were recruited through announcements and sign-up sheets on the WVU Department of Psychology subject pool bulletin board. Participants were offered both extra credit toward their grade in Introductory Psychology (or an upper level Psychology course that offered extra credit for participation in research protocols) and cash payment. Potential subjects were contacted by the experimenter and screened for the use of medication that affected cardiovascular functioning as well as general health status and their ability to bring a girlfriend/boyfriend of at least 2 months duration to one session (see Appendix A). Healthy individuals who did not use medications that affected cardiovascular functioning and had a boyfriend/girlfriend or husband/wife willing to accompany them to part of the session were invited to participate. Females selected to participate in the study were asked to report the date of the beginning of their last menstrual period so that test sessions could be scheduled with nine of the females being tested during their luteal phase and the other eight females being tested during their follicular phase. All selected participants were instructed to refrain from eating, smoking, using caffeine or alcohol, or exercising for 2 hours before the session. The modified SSQ was verbally administered at this time to obtain a baseline value of the perceived level of support participants received from their partners. Significant others were also offered payment as well as credit for their participation if they 
were taking Introductory Psychology or another psychology course that offered extra credit for participation in research protocols.

Upon arrival at the laboratory, informed consent was obtained, and demographic (see Appendix B; including measuring height and weight) and prescreening forms, the MSIS, and the FNE were completed. Procedures were explained to the participants and partners (e.g., participants were told that their partner would be joining them for one of two speeches they would deliver to offer social support that would help them during the task), and the impedance electrodes attached. A 16-minute adaptation baseline period was observed; during the final 6-minutes of this period impedance measures were continuously monitored and blood pressure was monitored every 2 minutes (on odd numbered minutes). In the Partner Present condition, during the adaptation phase, the partner was trained in another room how to be supportive during the task. Specifically, they were instructed to maintain an open body posture, make eye contact, nod, smile, and make the supportive verbal comments "yes, that's true" and "good point" quietly twice during the speech.

After the baseline adaptation period, the SAAC was administered to each participant to assess perceived stress during the rest period. Next, participants were given 4 minutes to prepare a speech on whichever speech topic they rated as most emotional (i.e., euthanasia, abortion, capital punishment). In the Partner Present condition, the partner was asked to read magazines in another room during the 4 minute preparation phase. The experimenter also left the participant alone during the preparation time, although cardiovascular system measures were obtained as described above.

At the end of the preparation time, in the Partner Present condition, the partner entered the room and was seated approximately 4 feet away, facing the participant. In both conditions, the 
experimenter, wearing a white laboratory coat, stood in the back of the room (approximately 4 feet away from the subject) making notes on a clipboard. A video camera, which was facing the subject, slightly to the subject's right, was focused on the subject during the 4-minute speech. At the end of the 4-minute speech, the camera was turned off and the participant was asked to complete the SAAC one more time, rating how he or she felt during the speech. In the Partner Present condition, the participant was asked to complete the modified SSQ to assess the participant's perception of the quality of support during the speech task. At the end of the first task, an identical procedure was followed for the alternate Partner Condition (i.e., if the first session was the Partner Present condition, the second session did not include the partner and vice versa). At the end of the second speech task, the subject was debriefed, and the participant and the partner were paid for their participation in the study.

Data Reduction. SBP and DBP readings were averaged for each rest phase using the three measurements obtained (i.e., readings at minutes 11,13 , and 15 of the first rest phase and minutes 1,3 , and 5 of the second rest phase). HR, stroke volume (SV), CO, and PEP were recorded continuously; measures for each parameter were averaged to obtain resting values for the respective rest phases for each parameter. Mean values during the 4-minute Speech Preparation phases and during the 4-minute Speech Tasks were calculated using an identical procedure. Body Surface Area (BSA) was calculated for each person using the equation BSA $=(\text { weight in kilograms })^{425} \mathrm{X}$ (height in centimeters $)^{.725} \mathrm{X}$ .007184 (Merck Manual (14th ed), 1982, p. 378) based upon measures obtained in the laboratory. By convention (e.g., Wilson, Lovallo, \& Pincomb, 1989), BSA is utilized to convert the volumetric measures of CO (i.e., the volume of blood ejected from the heart per minute) and SV (i.e., the volume of blood ejected from the heart in a single cardiac cycle) into cardiac index (CI) and stroke index (SI), 
respectively so that the comparability of these volumes is enhanced across individuals of varying body dimensions. For each rest, preparation, and speech period, mean arterial pressure was calculated using the formula MAP $=($ SBP - DBP $) / 3$ + DBP (e.g., see Allen et al., 1993). Likewise, TPR was calculated using the formula: TPR(in dyne/ $\mathrm{cm}^{5} /$ second $)=(\mathrm{MAP} / \mathrm{CO}) \mathrm{X} 80$ (e.g., see Sherwood, Dolan, \& Light, 1990). CI was calculated using the formula CI = CO/BSA (e.g., see Wilson, et al., 1989). SI was calculated using the formula SI = SV/BSA (e.g., see Wilson et al., 1989). Because relative changes in $\mathrm{CO}$ and $\mathrm{SV}$ measured by impedance cardiography have been shown to be more accurate than absolute measures of these parameters, CI, SI, and TPR were expressed as percent change from rest for each period.

\section{Results}

Because this study involved replication of earlier studies in basic methodology and an exploration of effects that existed beyond the basic methodology, these data were deemed to be exploratory in nature. Therefore, a significance level for all tests of $\underline{p}<.05$ was adopted for use in this study. This significance level can be justified for blood pressures and HR due to the confirmatory nature of the statistics. Also, because of the exploratory nature of this study, univariate analyses of variance were conducted on each dependent variable rather than conducting overall tests using multivariate models. In addition, effects sizes are reported to assist the reader in understanding the magnitude of the observed effect.

Demographic Variables.

One-way analyses of variance (ANOVAs) were performed on all continuous subject variables (i.e., age, height, weight, hours of sleep on the night before testing, duration of relationship with 
significant other, BSA, and years of education) and Pearson Chi-Square tests were conducted on family history of hypertension and student status to test for significant differences between males and females. Gender differences were noted for height, $\underline{\mathrm{F}}(1,32)=51.22, \underline{\mathrm{p}}<.001$, weight, $\underline{\mathrm{F}}(1,32)=$ 23.91, $\mathrm{p}<.001$, and BSA, $\underline{\mathrm{F}}(1,32)=45.91, \underline{\mathrm{p}}<.001$, with males being taller, heavier, and exhibiting a greater BSA than females (see Table 1). No other significant differences between genders were noted (all ps > .05). One-way ANOVAs on social questionnaire data (i.e., FNE, MSIS, and baseline SSQ6) also resulted in no significant differences between genders (i.e., ps $>.05$ ).

\section{$\underline{\text { Order Effects }}$}

To detect any order effects, a 2 X 2 [Order (Partner Present first, No Partner Present first) X Period (First chronological period, Second chronological period)] ANOVA was performed on each dependent variable for each rest, preparation, and speech period [i.e., SBP, DBP, all impedancederived measurements (i.e., HR, PEP, SV, CO, and TPR; see Table 2), and SAAC scores (see Table 3)]. The only significant Order X Period interaction was for DBP during the speech tasks, $\underline{\mathrm{F}}(1,32)=$ 4.73, $\underline{\mathrm{p}}<.05$ eta $^{2}=.163$. Participants who performed the speech task in the Partner Present condition first displayed a significantly higher $\mathrm{DBP}(\underline{\mathrm{M}}=89.9 \mathrm{~mm} \mathrm{Hg})$ than subjects who performed the speech task in the No Partner Present condition during their first speech $(\underline{\mathrm{M}}=84.1 \mathrm{~mm} \mathrm{Hg}$; see Figure 1). No other main effect for Order or Order X Period interactions were significant.

Significant main effects for Period were noted for SBP, HR, PEP, and SI. Higher SBP was observed during the second rest period $(\underline{\mathrm{M}}=117.7 \mathrm{~mm} \mathrm{Hg})$ as compared to the first rest period $(\underline{\mathrm{M}}=$ 113.7), $\underline{\mathrm{F}}(1,32)=12.54, \underline{\mathrm{p}}<.001, \mathrm{eta}^{2}=.28$. Resting HR was significantly lower during the first rest period $(\underline{\mathrm{M}}=72.9 \mathrm{bpm})$ in contrast to the second $(\underline{\mathrm{M}}=75.6 \mathrm{bpm})$ rest period, $\underline{\mathrm{F}}(1,32)=10.12$, 
$\underline{\mathrm{p}}<.01$ eta $^{2}=.24$; however HR was significantly higher during the first $(\underline{\mathrm{M}}=88.0 \mathrm{bpm})$ speech task in contrast to the second speech task $(\underline{\mathrm{M}}=83.8 \mathrm{bpm}), \underline{\mathrm{F}}(1,32)=5.48, \underline{\mathrm{p}}<.05$, eta $^{2}=.15$. Significant differences in PEP were observed between the first $(\underline{M}=103.7 \mathrm{~ms})$ and second $(\underline{M}=100.4 \mathrm{~ms})$ rest periods, $\underline{\mathrm{F}}(1,32)=7.51, \underline{\mathrm{p}}<.01$, eta ${ }^{2}=.19$, and the first $(\underline{\mathrm{M}}=92.3 \mathrm{~ms})$ and second $(\underline{\mathrm{M}}=96.3 \mathrm{~ms})$ speeches, $\underline{\mathrm{F}}(1,32)=5.79, \underline{\mathrm{p}}<.05$, eta ${ }^{2}=.15$. SI was significantly different between the first $(\underline{\mathrm{M}}=$

$\left.58.2 \mathrm{ml} / \mathrm{beat} / \mathrm{m}^{2}\right)$ and second $\left(\underline{\mathrm{M}}=56.2 \mathrm{ml} / \mathrm{beat} / \mathrm{m}^{2}\right)$ rest periods, $\underline{\mathrm{F}}(1,32)=6.16, \underline{\mathrm{p}}<.05$, eta $^{2}=.16$. No other Period effects were noted on any of the cardiovascular parameters (all ps $>.05$ ).

Regarding measures of self-reported arousal and stress, no significant main effects for Order or Period or Order X Period interaction were noted (i.e., all ps >.05). In sum, all participants exhibited a more relaxed cardiovascular system during the first rest period in contrast to the second rest period as evidenced by lower SBP and HR and longer PEP and greater SV. During the speech task, however, participants reacted somewhat more to the first speech task than the second speech task.

\section{$\underline{\text { Resting Cardiovascular Measures }}$}

A 2 [Gender (male, female)] X 2 [Partner Condition (Partner Present, No Partner Present)] ANOVA was performed on the rest period means for each cardiovascular parameter (i.e., SBP, DBP, HR, PEP, SI, CI, and TPR).

Regarding resting SBP, a significant Gender X Partner Condition interaction was observed, $\underline{\mathrm{F}}$ $(1,32)=5.67, \underline{p}<.05$, eta $^{2}=.15$. A significant main effect for Gender, $\underline{F}(1,32)=10.70, \underline{p}<.005$, $\operatorname{eta}^{2}=.25$, was observed as well but was not interpreted due to the significant interaction effect. Males exhibited higher resting SBPs $(\underline{\mathrm{M}}=121.1 \mathrm{~mm} \mathrm{Hg})$ than females $(\underline{\mathrm{M}}=110.3 \mathrm{~mm} \mathrm{Hg})$ across both rest periods but the difference was larger prior to the No Partner Present condition. Specifically, males in 
the No Partner Present condition displayed higher $(\underline{\mathrm{M}}=122.1 \mathrm{~mm} \mathrm{Hg})$ SBPs than females $(\underline{\mathrm{M}}=108.5$ $\mathrm{mm} \mathrm{Hg}), \underline{\mathrm{p}}<.0005$. Males in the Partner Present condition displayed higher $(\underline{\mathrm{M}}=120.0 \mathrm{~mm} \mathrm{Hg})$ SBPs than females $(\underline{\mathrm{M}}=112.1 \mathrm{~mm} \mathrm{Hg}), \underline{\mathrm{p}}<.05$. Also, females in the Partner Present condition exhibited higher resting SBPs $(\underline{\mathrm{M}}=112.1 \mathrm{~mm} \mathrm{Hg})$ than females in the No Partner Present condition $(\underline{\mathrm{M}}$ $=108.5 \mathrm{~mm} \mathrm{Hg}), \underline{\mathrm{t}}(17)=-2.21, \underline{\mathrm{p}}<.05$. No significant main effect was noted for Partner Condition.

Regarding resting HR, a significant main effect for Gender was observed, $\underline{\mathrm{F}}(1,32)=4.36, \underline{\mathrm{p}}<$ .05 eta $^{2}=.12$, with females exhibiting higher HRs $(\underline{\mathrm{M}}=77.8 \mathrm{bpm})$ than males $(\underline{\mathrm{M}}=70.7 \mathrm{bpm})$. Neither a significant main effect for Partner Condition nor a Gender X Partner Condition interaction was observed.

Regarding resting CI, a significant main effect for Gender was observed, $\underline{\mathrm{F}}(1,32)=10.23, \underline{p}<$ .005 , eta $^{2}=.24$, with females exhibiting higher CIs $\left(\underline{M}=4.81 / \mathrm{min} / \mathrm{m}^{2}\right)$ than males $\left(\underline{M}=3.71 / \mathrm{min} / \mathrm{m}^{2}\right)$. Neither a significant main effect for Partner Condition nor a Gender X Partner Condition interaction was observed.

Regarding resting TPR, a significant main effect for Gender was observed, $\underline{\mathrm{F}}(1,32)=7.98, \underline{\mathrm{p}}$ $<.01$, eta $^{2}=.20$, with males exhibiting higher TPRs $\left(\underline{\mathrm{M}}=2035.1 \mathrm{dyne} / \mathrm{cm}^{5} / \mathrm{sec}\right)$ than females $(\underline{\mathrm{M}}=$ $1541.0 \mathrm{dyne} / \mathrm{cm}^{5} / \mathrm{sec}$ ). Neither a significant main effect for Partner Condition nor a Gender X Partner Condition interaction was observed.

No resting significant main effects for Gender or Partner Condition or Gender X Partner Condition interaction effects were observed for resting DBP, PEP, or SI (i.e., all ps > .05). Measures of Cardiovascular Reactivity

Because some significant gender differences were observed regarding baseline levels of 
cardiovascular functioning, analyses of covariance (ANCOVAs) were used to analyze responses to the social manipulation. A 2 X 2 X 2 [Gender (male, female)] by [Partner Condition (Partner Present, No Partner Present)] by [Phase (Preparation, Speech Task)] mixed factors ANCOVA, with both Partner Condition and Phase serving as repeated measures, was performed on each cardiovascular parameter. Parameters tested included SBP, DBP, and all impedance-derived measurements (i.e., HR, PEP, SI\% change, $\mathrm{CI} \%$ change, and TPR\% change scores), with respective resting measures serving as covariates for SBP, DBP, HR, and PEP. Because SI\%, CI\%, and TPR\% change scores reflect change relative to resting measures, no covariate was utilized for these parameters. The word "phase" is utilized to distinguish the different active phases of the study (i.e., preparation and speech) from rest periods.

Regarding SBP response, a significant main effect was observed for Phase, $\underline{\mathrm{F}}(1,32)=102.41$, $\underline{p}<.001$ eta $^{2}=.76$, with a higher SBP reactivity observed during the Speech Task phases (adjusted $\underline{\mathrm{M}}=132.0 \mathrm{~mm} \mathrm{Hg}$ ) than during the Speech Preparation phases (adjusted $\underline{\mathrm{M}}=119.7 \mathrm{~mm} \mathrm{Hg}$ ). No significant main effects for Gender or Partner Condition were observed (all ps > .05). Likewise, no significant interactions were observed (i.e., all ps > .05).

Regarding DBP response, a significant main effect was noted for Phase, $\underline{F}(1,32)=30.46, \underline{p}<$ .001 eta $^{2}=.49$. Higher DBP responses were observed during the Speech Task phases (adjusted $\underline{\mathrm{M}}=$ $86.2 \mathrm{~mm} \mathrm{Hg}$ ) than during their respective Speech Preparation phases (adjusted $\underline{\mathrm{M}}=78.7 \mathrm{~mm} \mathrm{Hg}$ ). No other main effects or interactions were significant, although the Partner Condition X Phase interaction approached significance $(\mathrm{p}=.06)$, with DBPs during the Partner Present Condition somewhat higher $(\underline{\mathrm{M}}=83.6 \mathrm{~mm} \mathrm{Hg})$ than DBPs during the No Partner Present Condition $(\underline{\mathrm{M}}=81.4 \mathrm{~mm} \mathrm{Hg})$, but only 
during the speech task (i.e., not during the preparation phase). Thus, participant's blood pressures, both SBP and DBP, were observed to increase between the preparation phase and the speech task (Uchino et al., 1996).

Regarding HR response (see Figure 2), a significant Gender X Phase interaction was noted, $\underline{\mathrm{F}}$ $(1,32)=4.44, \underline{p}=.04$, eta $^{2}=.30$. Although both males and females exhibited increased HR during the Speech tasks (i.e., over their respective Preparation phases), females displayed a greater increase in HR (adjusted $\underline{\mathrm{M}}=90.8 \mathrm{bpm}$ ) than males (adjusted $\underline{\mathrm{M}}=81.0 \mathrm{bpm}$ ) during the Speech tasks. A significant main effect for Phase, $\underline{\mathrm{F}}(1,31)=13.53, \underline{\mathrm{p}}=.001, \mathrm{eta}^{2}=.30($ Speech higher than Preparatory Phase) was not interpreted due to the significant interaction effect involving this variable. No significant main effect for Partner Condition was observed (i.e., $p>.05$ ). No other interactions were significant (i.e., all ps $>.05$ ). Similar to the blood pressure increases discussed, HRs were observed to increase in response to the speech task, particularly in females.

Regarding PEP response (see Figure 3), a significant Gender X Phase interaction effect was detected, $\underline{\mathrm{F}}(1,32)=4.42, \underline{\mathrm{p}}<.05$, eta $^{2}=.12$. Although males (adjusted $\left.\underline{\mathrm{M}}=98.7 \mathrm{~ms}\right)$ and females (adjusted $\underline{\mathrm{M}}=98.5 \mathrm{~ms}$ ) displayed comparable PEP responses during Preparation, females exhibited a significantly lower PEP (adjusted $\underline{\mathrm{M}}=91.3 \mathrm{~ms}$ ) than males (adjusted $\underline{\mathrm{M}}=97.3 \mathrm{~ms}$ ) during the Speech task. A significant main effect for Phase, $\underline{\mathrm{F}}(1,32)=9.87, \underline{\mathrm{p}}=.004$ eta $^{2}=.24$, was also observed but was not interpreted due to the significant interaction effect involving this variable. No significant main effects for Partner Condition or Gender were observed (i.e., ps > .05). No other interactions were significant (i.e., all ps > .05).

Regarding SI percent change, a significant main effect for Gender was observed, $\underline{\mathrm{F}}(1,32)=$ 
$6.70, \underline{\mathrm{p}}<.05$, eta $^{2}=.31$, with males displaying a larger decrease $(\underline{\mathrm{M}}=-10.91 \%)$ in SI than females $(\underline{M}=-4.37 \%)$ during the speech and speech preparation phases. No significant main effects for Partner Condition or Phase were observed (i.e., ps > .05). No interactions were significant (i.e., all ps $>.05)$.

Regarding CI percent change, a significant Gender X Phase interaction effect was observed, $\underline{\mathrm{F}}$ $(1,32)=5.24, \underline{p}<.05$, eta $^{2}=.141$. Females displayed significantly greater CI reactivity $(\underline{\mathrm{M}}=+12.27$ $\%)$ to the speech tasks than males $(\underline{M}=-1.71 \%)$. Significant Main effects for Gender and Phase were also observed but were not interpreted due to the interaction effect involving these two variables. No main effect for Partner Condition or other interaction effects were significant (i.e., all ps $>.05$; see Figure 4).

Regarding TPR percent change scores, a significant interaction effect was noted for Gender X Phase, $\underline{\mathrm{F}}(1,32)=9.17, \underline{\mathrm{p}}=.005$ eta $^{2}=.22$. Males displayed significantly greater $(\underline{\mathrm{M}}=23.65 \%) \mathrm{TPR}$ reactivity to the speech tasks than females $(\underline{\mathrm{M}}=4.92 \%$; see Figure 8$)$. In addition, significant main effects for Gender and Phase were observed but were not interpreted due to the significant interaction effect involving these variables. No significant main effect for Partner Condition or other interactions were observed (i.e., all ps $>$.05). In summary, males in this study responded with less myocardial change and more peripherally vascular change than females to the speech tasks.

\section{$\underline{\text { Stress, Arousal, and Support Measures }}$}

A 2 X 2 [Gender X Partner Condition] ANOVA was conducted on both the Stress and Arousal subscales of the SAAC. A significant main effect for Gender was observed on the Stress subscale, $\underline{\mathrm{F}}(1,32)=6.40, \underline{\mathrm{p}}<.05$. Females scored significantly lower $(\underline{\mathrm{M}}=26.62)$ than males $(\underline{\mathrm{M}}=$ 
31.62), indicating that females reported less stress than males during both speeches. Females reported somewhat less arousal as a result of the speech tasks than males as well, but the difference was not significant. No significant Partner Condition main effects or Gender X Partner Condition interaction effects were observed for SAAC Stress or Arousal subscale scores (i.e., all ps > .05).

Regarding scores on the modified SSQ6 after the Partner Present speech task, a one-way ANOVA revealed that females reported significantly lower levels $(\underline{M}=28.76)$ of perceived support from their partners, $\underline{\mathrm{F}}(1,32)=7.12, \underline{\mathrm{p}}<.02$ eta $^{2}=.18$, than males $(\underline{\mathrm{M}}=32.82)$.

\section{$\underline{\text { Correlational Analyses }}$}

To examine correspondence between self-reported quality of relationship and degree of fear of negative evaluation with cardiovascular reactions to the speech task, scores on the MSIS and the FNE were correlated with difference scores (i.e., Speech - Baseline) for all cardiovascular parameters (i.e., SBP, DBP, HR, PEP, SI, CI, and TPR), using partial correlations removing the effect of respective baseline values. No significant partial correlations were noted (i.e., all ps $>.05$; see Table 4), suggesting that there were no direct relations between perceived quality of relationship or negative fear of evaluation and cardiovascular reactivity to the speech task utilized in this study.

\section{Hemodynamic Profiles}

Finally, individuals were classified as CI reactors, TPR reactors, or mixed reactors based on criteria established by Kasprowicz, Manuck, Malkoff, and Krantz (1990). That is, all subjects whose increase in CI was at least one standard deviation above the mean and whose TPR was less than the mean of all subjects during at least one speech were categorized as CI reactors. Conversely, subjects whose increase in TPR was at least one standard deviation above the mean and 
whose increase in CI on either speech task was less than the mean of all subjects during at least one speech were categorized as TPR reactors. Mixed reactors were defined as individuals who displayed an increase in both CI and TPR during the task and the difference between the CI and TPR difference was within one standard deviation of the mean (see Kasprowicz et al., 1990 for details). Persons who were identified as CI, TPR, or mixed reactors in at least one task were classified by the appropriate label. No subjects displayed contrary reactor status between tasks (i.e., none were CI reactors during one speech and TPR reactors during the other speech). Six CI reactors ( 5 females, 1 male) were identified by these methods. In addition, six TPR reactors ( 5 males, 1 female) were identified. One male was classified as a mixed reactor by the above criteria. A Pearson Chi Square analysis was conducted on these data, excluding the mixed reactor (due to an expected cell value less than 1), and a significant Chi-square $(1)=5.33, \underline{p}<.05,8=.67$, was noted, indicating that the frequency of females versus males being myocardially reactive versus TPR reactive was significantly different.

\section{Discussion}

The present study had two purposes. The first was to examine the specific cardiovascular mechanisms responsible for the HR and/or blood pressure attenuation effect of social support on cardiovascular reactivity to a task. This effect typically has been observed when the task is performed in the presence of a supportive friend as opposed to alone or in the presence of a neutral experimenter. Unfortunately, in this experiment, no attenuation effect of social support was observed, rendering conclusions regarding the effect of social support on hemodynamic functioning meaningless. The second purpose of this study was to examine whether cardiovascular reactivity to a speech task was differentially affected by social support among males and females. Because no effect for social support 
was observed, the questions pertaining to how male and female participants respond to social support also were unanswered.

Because previous literature has reliably observed an attenuating effect for social support, the reason that no social support effects were observed during this study must be examined. This study was designed to replicate certain aspects of previous studies and to extend the finding of these studies, largely through employing impedance cardiography to provide hemodynamic information in addition to HR and blood pressure information. Because we believed that social support required a social task (e.g., giving a speech) for realistic examination, ecological validity was a major concern in the design of this study. In considering a speech task, although the importance of the evaluative component identified by Kamarck et al. (1990) was considered, it was believed that, due to the nature of social interactions (i.e., most significant others can hear and observe their partner's activities and are in an evaluative position), it was important to allow partners to listen to the speech and interact with his or her partner. As a result, this study aimed to replicate studies that utilized comparable social tasks (i.e., Christenfeld et al., 1997; Gerin et al.,1992; Kleinke \& Williams, 1993; Lepore, 1995; Lepore et al., 1993; Uchino \& Garvey, 1997). Despite this task being largely an extended replication of earlier studies (e.g., Christenfeld et al., 1997; Lepore et al., 1992), the effects observed in those studies were not replicated in this study.

Comparing the current study with specific studies in this body of literature, Gerin et at. (1992) observed an attenuating effect of social support during a verbal task in which a female participant was verbally attacked during discussion of a controversial issue. Inconsistent with the present study, however, Gerin used an implied threat in their study design (i.e., being verbally "attacked") and social 
support was provided by confederates of the experimenter rather than acquaintances or partners of the participant.

The findings of the present study were consistent with the work conducted by Kleinke and Williams (1993), who also failed to observe an attenuating effect of social support. Their social manipulation (i.e., 2-second touch), however, was much less overt than the trained partners utilized in the current project. Also, due to the inconsistency concerning the importance of "touch" in eliciting an effect for social support, we did not employ touch as part of our socially supportive manipulation. Regardless, both studies failed to observe an attenuated cardiovascular reactivity to stress with social support.

Although the task and procedure in Lepore et al.'s $(1993,1995)$ studies were relatively similar to those employed in the current study, the results were not consistent. However, some differences in procedure between Lepore et al.'s studies and the current study exist. For example, Lepore et al. utilized same-gender confederates and all social support was provided by a confederate rather than an acquaintance or partner of the participant.

Although Uchino and Garvey (1997) observed a significant difference between groups (i.e., the group that was told that support was available displayed attenuated cardiovascular reactivity to the speech task compared to the group that did not have support available), their procedure differed substantially from the procedure utilized in this study. Because no support was actually given, there are too many procedural differences to justify a direct comparison of the results between their study and the current study.

Among previous studies that have examined the effects of social support on cardiovascular 
reactivity to a task, the procedure employed by Christenfeld et al. (1997) is most similar to the procedure utilized in the current study. Christenfeld et al. utilized a speech task and manipulated social support from the participant's friend versus a confederate of the experimenter. The support person was able to observe and potentially evaluate the performance of the participant. However, they utilized a between-subjects design and only female participants in their protocol. Despite the procedural similarities between these two studies, Christenfeld et al. observed an attenuating effect of social support, unlike the results observed in the current study. The difference in the gender of the samples employed cannot explain the discrepant results because, even within the females in the current study, no effects for social support were observed. Use of a supportive friend versus a supportive partner, however, may help explain the differences between the current study and previous work.

The failure of the current study to observe an attenuating effect that was observed in previous studies utilizing comparable methodology is similar to the contrary effects obtained by Edens et al. (1992), who obtained differential findings in a different laboratory setting utilizing a similar protocol (i.e., Kamarck et al., 1990). Edens et al. attributed their lack of a significant finding to the more informal laboratory atmosphere versus the more formal setting employed by Kamarck et al (1990). The same mechanism may be operative in this versus earlier studies, in that the same, more informal, atmosphere utilized by Edens et al. was the setting for this study. As in the Edens et al. study, this study was conducted by graduate students rather than by a professor, as was the case in the Kamarck et al. (1990) study. In an attempt to maximize formality in the current study, both graduate students were dressed professionally (i.e., in dress clothes), wore lab coats, and maintained a detached air during the speech tasks. However, the more informal ambiance of a university in a more rural setting (versus a 
more urban setting) could not be changed.

Interestingly, in an attempt to explain their discrepant results, Edens et al. (1992) noted that the HR and blood pressures observed in their study were outside the range of the HR and blood pressure increases obtained in the previous studies, despite task similarity. Specifically, studies prior to and including Kamarck et al. (1990) observed Alone HR increases ranging from 11.5 to 17.8 bpm, whereas Edens et al. obtained only a 9.1 bpm increase in their study. Similarly, previous studies observed SBP and DBP response increases ranging from 18.7 to $20.7 \mathrm{~mm} \mathrm{Hg}$ versus a $14.5 \mathrm{~mm} \mathrm{Hg}$ increase in SBP and an $8.9 \mathrm{~mm} \mathrm{Hg}$ increase in DBP observed by Edens et al. Although, in the current study, the task was of a different nature (i.e., speech rather than a math task) than in the previous studies, the increase in HR during the Alone condition was $11.5 \mathrm{bpm}$, which is within the range observed by researchers prior to Edens et al. However, the increases in SBP and DBP in the current study were 16.01 and $10.39 \mathrm{~mm} \mathrm{Hg}$, respectively. These blood pressure responses are somewhat lower than those observed by Kamarck et al. and previous researchers, but not as low as those measures observed by Edens et al. Therefore, it is unlikely that the lower HR or blood pressure reactions observed in the current study can account entirely for the nonsignificant results.

Kamarck, Peterman, \& Raynor (1998), in a review of both published and unpublished studies performed in their laboratory, found that studies that utilized a male experimenter produced significant attenuation effects and their studies that utilized a female experimenter yielded nonsignificant results and small effect sizes (i.e., did not attain a significant attenuating effect for social support). Although the experimenter in the room with female participants in the current study was a male, the principal investigator, who was present for every participant's session, was a female. This gender-of- 
experimenter effect may be part of the reason that no attenuating effect for social support was observed in this study and may also help to explain why these results are inconsistent with much of the previously published literature in this field. However, it must be noted that other studies that utilized female confederates (e.g., Christenfeld et al., 1997) have observed effects for social support.

Regarding the use of opposite-gender experimenters, Thorsteinsson, James, and Gregg (1998) suggested that the gender incongruence in the Kamarck et al. (1990) study (i.e., male experimenter with female participants) may partially explain why Kamarck et al. observed a significant attenuating effect of social support whereas Edens et al. (1992) did not observe the same attenuating effect because they utilized a female experimenter with female participants. Because the current study employed an opposite-gender experimenter design and did not obtain a significant attenuating effect resulting from social support, it is unlikely that the explanation offered by Thorsteinsson et al. is a major factor in determining whether an attenuating effect is or is not observed as a result of a social support manipulation.

Another factor that may be influential in explaining these discrepant findings is perceived control (Seligman, 1972). Perceived control by participants may lessen the physiological impact of the tasks, given that individuals in the current study chose whether or not to participate in this research protocol and were provided with alternate methods for obtaining extra credit in their class. At the University of Pittsburgh, where Kamarck et al. $(1990,1995)$ conducted their studies, all introductory psychology students are required to either participate in a study or write a reaction paper. Participants in that setting may feel more "coerced" into participation and completing the study. The increased perceived control students at WVU experience may result in lower cardiovascular reactions to stress and lower 
opportunity to exhibit the attenuating effect of social support.

An additional factor that may be important in understanding the lack of attenuating effect obtained in this study is the evaluative component addressed by Kamarck et al. (1990). Because significant others were seated directly in front of the participants, approximately four feet away, they were in the position to observe and evaluate the performance of the participant. Further, in a metaanalysis examining the verbalization of support during tasks (in addition to the evaluative component), Kamarck et al. (1998) found that there may be a cardiovascular response-enhancing effect that resulted from active verbal support, contrary to effects observed by Christenfeld et al. (1998) and Gerin et al. (1992). In the current study, verbalizations were modeled after those utilized in the Gerin et al. study which, according to Kamarck, may have elicited more social evaluation than social support.

Although no other published study examining cardiovascular reactivity to a speech task employing significant others has been conducted, other studies that have examined the effects of social support have utilized significant others (e.g., Kirschbaum, Klauer, Filipp, \& Hellhammer, 1995). In the Kirchbaum et al. study, attenuated cortisol levels in males were observed when support was provided by a stranger, with even higher levels of attenuation attained when support was provided by significant others. Interestingly, however, no attenuating effect was observed in female participants; cortisol levels were actually higher in the Partner Support condition versus the No Support and Stranger Support conditions. Although the current study did not obtain an attenuating effect in males, the equivocal results of Kirschbaum et al. suggests the use of significant others may impact experimental findings, particularly when female participants are used because females do not appear to respond to social support from their partners with as large or as consistent an effect as do males. 
An alternate explanation for the results of this study is that the attenuating effects are not, in fact, present when a task is performed in the presence of one's significant other (i.e., the null hypothesis is true). Butler and Baumeister (1998) conducted a study that examined performance as the outcome measure where they manipulated perceived social support during a math task from the audience. Participants were told whether her or his friend or a confederate was observing their performance from behind a one-way glass. Because they could not see who was providing the support and no friends actually observed the performance, their social support condition manipulated perception of support rather than actual support. Although they did not measure physiological reactivity to the math task in this study, they observed that participants performed more poorly in the perceived presence of a friend than in the perceived presence of a neutral confederate. Butler and Baumeister suggested that expecting to have future interactions with a person who is in an evaluative position (e.g., observing their performance during a task) may increase pressure to perform well. Although there are obvious differences between the methods employed by Butler and Baumeister and the current study (e.g., use of a math task versus a speech task, perceived support versus actual support, support from a friend versus a significant other), it is also possible that observation by a partner may interfere with optimal performance and possibly elevate psychophysiologic reactions.

Also, the Butler and Baumeister (1998) study raises the question of the importance of perceived support, which may be salient in the current study. The procedure employed in this study involved testing all participants in both conditions on the same day, which is different from other studies in this body of literature. For example in the Kamarck et al., 1990; 1995 studies, participants who were tested in the Alone condition were not accompanied to the laboratory by a friend. Therefore, 
they neither had perceived nor enacted support. Conversely, participants who were accompanied to the laboratory by a friend had both perceived and enacted support. In the current study, however, because both Partner Conditions were conducted on the same day (and all partners waited in the next room for their significant other to complete the protocol), our participants during the No Partner Present condition did not have enacted support because their significant others were not in the room. They, however, may have felt that they had perceived support because they knew that their significant others were waiting for them and available to them should the need arise; support was available to them whereas it was not to Kamarck's comparable (i.e., Alone) participants. Similar to the Kamarck et al. studies, participants in the Partner Present condition in the current study had both perceived and enacted support from their significant others. The perceived support that participants may have felt during the No Partner Present condition of the current study may have attenuated their cardiovascular parameters to a degree sufficient to obscure any attenuating effect present due to the presence of the partner during the Partner Present condition. Thus, the difference may be explained by a procedural difference rather than a difference between a within- versus a between-subjects design.

An additional explanation for the lack of a positive effect for social support in this study pertains to social pressure. That is, pressure to perform well may result from receiving social support from an acquaintance (with whom one expects to have an on-going relationship) versus a confederate (whom the participant will probably never see again). So, perhaps an attenuating effect for social support exists only if the participant does not feel increased pressure to perform well (e.g., the evaluative component is removed as in Kamarck et al. (1990)). In studies such as the current study, where there was a potential evaluative component that increased the "pressure" on participants to perform well, the 
"pressure factor" may have acted to negate any attenuating effect that existed due to social support.

We made an effort in this study to control for many extraneous variables that could affect cardiovascular reactivity to stress such as family history of hypertension; food, caffeine, and tobacco intake; menstrual status; medications; and exercise. We were also careful to recruit subjects who were similar in age, student status, years of education, duration of their current relationship, and hours of sleep the night before their participation in the study. None of these parameters was different between groups. Because other researchers generally have failed to control for these factors, their findings may be related to one of these unmeasured factors and its relation to reactivity to a task.

In addition, we measured several cognitive and individual parameters that may have helped understand differences that may have accounted for the attenuating effect of social support. We utilized the FNE to monitor the amount of fear of negative evaluation - or loosely speaking social anxiety - that could affect stress associated with the task. No significant differences were observed. The MSIS was used to measure "quality" of the relationship under the assumption that poorer relationships may impact the quality of support received as well as cardiovascular reactivity to the speech task. Again, no difference existed between groups, nor were either of these instruments correlated with any cardiovascular parameter measured in this study.

\section{Gender Differences in Cardiovascular and Hemodynamic Response to Stress}

Although the primary questions of this investigation were not answered, the current study can contribute to the literature examining gender differences in hemodynamic response to stress. Regarding gender effects, females in this study exhibited higher resting HRs and CIs than males. The presence of higher HRs in females has been well-documented in the literature (Saab, 1989). 
Conversely, males displayed higher resting SBPs and TPRs than females in the current study. The presence of higher resting SBP in males 18 to 44 years old has been obtained from data from the Health and Nutrition Examination Survey of 1971 - 1974 (Saab, 1989). Higher resting TPRs among males have been less reliably reported in the literature. Regarding cardiovascular reactivity to the speech tasks, females exhibited higher HR, SI, and CI reactivity to the speech task than males. Males displayed longer PEP responses (suggesting less \$-adrenergic responsivity among males than females) and higher TPR percent change scores than females. Interestingly, despite resting SBP being higher among males than females, blood pressure reactivity to the task was not greater among males than females.

Other studies have examined gender differences between males and females on both hemodynamic and blood pressure measures, with equivocal results. For example, Lawler, Wilcox, and Andersen (1995) examined cardiovascular reactivity (including blood pressure and impedance cardiograph measures) to mental arithmetic, video game tasks, and an anger recall interview. Although they observed higher resting SBP among males (as was observed in this study), they also reported that males exhibited higher cardiac output responses to the various challenges than females, which was not observed in this study. They categorized their subjects as myocardially reactive or peripherally reactive, using the same criteria utilized in this study.

Contrary to the current results, males were more likely to be categorized as cardiac reactors and females as vascular reactors. Using an identical procedure, they were able to categorize $22 \%$ of their sample as either myocardially or vascularly reactive versus $35 \%$ of the participants in this study sample. The number of males and females categorized as either cardiac output or vascular reactors 
were not significantly different in their study, whereas there was a significant difference between groups in the current study. Significantly more males than females were categorized as vascular reactors and significantly more females than males were categorized as cardiac output reactors.

Allen, Stoney, Owens, and Matthews (1993) also utilized impedance cardiography to examine gender differences in cardiovascular reactivity to laboratory tasks (i.e., math, mirror tracing, Stroop color word, and isometric handgrip tasks). In contrast to the results observed by Lawler et al. (1995), Allen et al. concluded that males were more likely to be categorized as vascular reactors and females were more likely to be categorized as myocardial reactors to a subset of tasks. Although Allen et al. categorized myocardial and vascular reactors according to slightly different criteria than either Lawler et al. or the current study, they found that more males were classified as vascular reactors and more females classified as myocardial reactors, as was observed in the current study.

Both Girdler et al. (1990) and Hurwitz et al. (1993) examined cardiovascular and hemodynamic reactivity to a speech task utilizing impedance cardiography with both male and female participants. However, no social support manipulation was included in their protocols. Participants in the Girdler et al. study performed two different speech tasks. Girdler et al. observed that females were more myocardial reactors and males were more vascular reactors during the recall speech task only (i.e., no gender difference was observed during the speech that involved an evaluative component). Hurwitz et al. employed a speech task comparable to the task utilized by Girdler et al. and, like Hurwitz et al., they observed that males and females displayed similar responses to the evaluative speech task (i.e., no significant difference was observed). It is apparent from these equivocal results that many aspects of the task utilized to categorize myocardial versus peripheral reactors are critical to the 
outcome of the categorization process. For example, Lawler et al. (1995) utilized a nonverbal math task and observed that two thirds of their myocardial reactors were males versus two thirds of their peripheral reactors being female, although the difference was not significant. Allen et al. (1993) categorized their participants as myocardial (i.e., increased HR) or peripheral reactors on math mirror tracing, Stroop Color-Word, and isometric hand grip tasks. They observed that significantly more females than males were myocardial reactors on the mirror tracing task only, with a trend toward this result for the Stroop task. No significant differences were observed for HR between males and females for the math or handgrip tasks. Conversely, they observed that significantly more males than females were peripheral reactors on the Stroop and handgrip tasks. No significant differences were observed for TPR between males and females for the math or mirror tracing tasks. Comparing the results of the math task between Allen et al. and Lawler et al. indicate divergent results. Specifically, the myocardial reactor group in the Lawler et al. study for the math task included 7 males and 4 females, whereas the myocardial reactor group in the Allen et al. study was composed of 4 males and 10 females.

Conversely, the peripheral reactor group for the math task in the Lawler et al. study included 5 males and 10 females, whereas the peripheral reactor group in the Allen et al. study was composed of 8 males and 6 females. Therefore, type of task may not be the salient factor to explain these divergent results. Because no math task was performed in the current study, the results of this study cannot be compared directly to the findings of Lawler et al. or Allen et al.

\section{Subjective Responses to the Speech Task}

Regarding SAAC scores, consistent with the results obtained by as Lepore (1995), perceived stress appeared to distinguish cardiovascular reactivity to the speech task utilized in this study between 
gender groups, whereas perceived arousal did not differentiate between groups. Lepore interpreted this result as suggesting that individuals are not good judges of the degree of their physiological arousal. However, males in the current study reported experiencing significantly more stress and marginally more arousal than females as a result of the task. This finding is contrary to Lepore's hypothesis regarding individuals' ability to judge their physiological arousal and suggests that males may be better judges of their stress and/or arousal than females. This is particularly true in the present study where females exhibited greater HR, PEP, SI, and CI reactions to the speech yet evidenced less self-reported stress and arousal. It is possible that because males exhibited greater TPR response to the speech than females that their increased ratings of stress and arousal corresponded more closely to the " adrenergic-mediated TPR response than the \$-adrenergic-mediated cardiac response. Regardless of the reason for these findings, they are quite inconsistent with a literature that demonstrates males underreport affective states in comparison to females (Uchino et al., 1996).

Gender effects were also observed on the SSQ6 administered after the Partner Present speech task. Although there was no difference between genders on their baseline perception of support received from their partners, after the speech in the Partner Present condition, females in this study reported receiving lower levels of perceived support from their partners than males. Although no previous studies have utilized the SSQ6 in this way, evidence that females benefit less from social support than males has been observed (e.g., Kirchbaum et al., 1995). In the current study, females did not display the exaggerated physiological response as did the females in the Kirchbaum et al. study, although they did report subjective knowledge of a qualitative lack of support compared with their male counterparts. Kirchbaum et al. alluded to the "boomerang effect" (p. 29) of social support to explain 
the unexpected increased magnitude of cortisol reactivity among their female participants. According to this hypothesis females, expecting social support, perceive social evaluation instead, leading to increased cardiovascular arousal. The boomerang effect may be operative in the current study as well. Females in this study may not have perceived that social support offered by their significant other as helpful, but rather as evaluative or judgmental. A possible explanation for this perception may be that females traditionally respond to affective support whereas males respond to more instrumental support. Because of the instrumental nature of the support scripted in this study (i.e., showing that the partner was paying attention by saying, "yes, that's true" and "good point" rather than showing emotional support), males may have felt more comfortable than females with the quality of support provided. Limitations of this Study

Despite the fact that this study was largely a replication and extension of previous studies that addressed the effects of social support on cardiovascular reactivity to a mental challenge, some procedural flaws in the design of the study may have prevented observation of the expected results. Regarding order effects, differences between values from Rest periods 1 and 2 were observed for HR, PEP, and SI, which suggested that the 5-min rest period allowed between tasks for cardiovascular parameters to return to baseline was not sufficient.

The SAAC, which was utilized in this study to measure perceived stress and arousal, was administered after the speeches, and thus suffers from being not only self-report, but also retrospective in nature. Better methods of assessing cognitive arousal and stress experienced during a task should be pursued (e.g., measuring during the task by direct behavioral observation).

In retrospect, this study would have benefitted from measuring task performance (e.g., see 
Christenfeld et al., 1997). These data may have been useful in determining whether an evaluative component impaired performance (e.g., such as that proposed by Butler and Baumeister, 1998) and was a significant factor in the effects observed from the social support manipulation. These data may have also helped explain alternate influential factors in this study (e.g., behavioral changes, verbal behaviors).

Lastly, in order to identify specific factors that were operative in this study that contributed to nonsignificant attenuation effects in comparison to other studies that have observed significant differences, an exact replication of previous studies may have been warranted. Because we changed so many procedural elements in this study, we are left with inconsistent findings that are not easily interpreted. For example, compared with the Christenfeld et al. (1997) study, the discrepant results may be attributed to the location of the experimenter (i.e., behind the participant in the Christenfeld et al. study, in front of the participant in the current study); use of a friend (by Christenfeld et al.) versus a significant other (in this study); using a same-gender experimenter (in the Christenfeld et al. study) versus an opposite-gender experimenter (in the current study); or being fitted with a Finapres finger cuff to measure blood pressure (in the Christenfeld et al. study) versus being fitted with impedance electrodes and a traditional blood pressure cuff (in the current study). Perhaps altering the conditions of the experiment in a more controlled way would have enabled us to interpret our findings more completely.

\section{$\underline{\text { Future Directions }}$}

This study failed to replicate the original Kamarck et al. (1990) findings, much as Edens et al. (1991) and Sheffield and Carroll (1994) failed to replicate these results. In some ways, this study may 
have had more ecological validity than the Kamarck et al. study because (a) the nature of the task was more social in nature (i.e., a speech task versus mental arithmetic and concept formation tasks), and (b) because the person providing social support was in an evaluative position. Despite the failure to replicate previous findings, the attenuation effect observed as a result of social support appears to be evident in several studies. However, it appears to be operative only at specific times, which appear to be a function of the degree of evaluation present and threat inherent in the specific laboratory protocol (i.e., social support may act as a stress buffer). Anecdotal knowledge states that "there is safety in numbers," which may indicate that when one is threatened, social support has a kind of "bystander effect," where responsibility for action is shared, or diffused. However, when one is alone, one's own system must mobilize to face the threat, demanding additional sympathetic nervous system activation, which translates in cardiovascular terms to a higher (i.e., not attenuated) cardiovascular system activation. It may be of interest to monitor not only the participant during a speech task, but also the partner, to observe if both display increased reactivity, and perhaps "share" the load. It is also possible that social support does not "attenuate" cardiovascular reactivity; perhaps the situation demands, given the presence of an ally, are just less.

It would be interesting if future studies would compare the effects of social support by friends versus romantic partners of the participants to observe whether the quality of the relationship differentially impacts cardiovascular and hemodynamic variables. Allen et al. (1991) compared pet dogs to friends and observed that the presence of pets attenuated the cardiovascular response to a task more than friends. One may hypothesize, due to the "boomerang effect" and the increased evaluative component observed in significant other studies, that the presence of friends may be more effective in 
attenuating the cardiovascular response to a task than the presence of a mate. It is also interesting to speculate why partners may not provide as effective support as well as friends and seek to identify these processes (e.g., increased perceived evaluation between significant others, perhaps due to a desire to impress the partner) and incorporate their evaluation into the experimental protocol.

Another issue that deserves attention in this body of literature involves the number of studies that are performed but are not published. As stated in Kamarck et al. (1998), these experimenters have performed eight studies and have obtained significant results in only three of these studies. Perhaps more summary articles such as this recent work by Kamarck et al., outlining both significant and nonsignificant findings, may assist other researchers addressing the same question to identify specific parameters that may affect the salience of social support to cardiovascular reactivity to tasks. This would also prevent future researchers from having a "skewed" interpretation of this body of literature. In retrospect, decreasing the evaluative component by the significant other, as was done by Edens et al. (1992) and Kamarck et al. (1990; 1995), and increasing the threat (e.g., have the experimenter stand 1 or 2 feet away and wear a white laboratory coat) may have made the difference between significant results and nonsignificant results for this study. However, the question of ecological validity remains salient; "What represents real life and how can we best study these real effects in the laboratory?" is really the more pertinent question.

As stated in the introduction of this paper, social support has been reliably and prospectively associated with mortality and cardiovascular morbidity (e.g., House et al., 1988). A variety of underlying psychological and biological mechanisms have been proposed in an attempt to understand precisely how social support enhances life and health (e.g., see Cohen, Kaplan, \& Manuck, 1994). 
Although many mechanisms have been proposed, none has been identified definitively that can account for the variety of effects observed in this body of literature. However, despite the failure of this study to observe an attenuating effect of social support on cardiovascular reactivity to a task, the body of literature that has observed the salubrious effect of social support is extensive. The study of social support as a life- and health-enhancing mechanism should continue so that the operative aspects of social support can be identified and employed to enhance health and prolong life. 


\section{References}

Allen, K. M., Blascovich, J., Tomaka, J., \& Kelsey, R. M. (1991). Presence of human friends and pet dogs as moderators of autonomic responses to stress in women. Social Psychology, 61, 582-589.

Allen, M. T., Stoney, C. M., Owens, J. F., \& Matthews, K. A. (1993). Hemodynamic adjustments to laboratory stress: The influence of gender and personality. Psychosomatic Medicine, 55, 505-517.

Andersson, L. (1985). Intervention against loneliness in a group of elderly women: An impact evaluation. Social Science and Medicine, 20, 355-364.

Berkman, L. F., \& Syme, S.L. (1979). Social networks, host resistance, and mortality: A nine year follow-up study of Alameda County residents. American Journal of Epidemiology, 109, 186-204.

Bland, S. H., Krough, V., Winkelstein, W., \& Trevisan, M. (1991). Social network and blood pressure: A population study. Psychosomatic Medicine, 53, 598-607.

Butler, J., \& Baumeister, R. (1998). The trouble with friendly faces: Skilled performance with a supportive audience. Journal of Personality and Social Psychology, 75, 1213-1230.

Cacioppo, J. T., Berntson, G. G., Binkley, P. F., Quigley, K. S., Uchino, B. N., \& Fieldstone, A. (1994). Autonomic cardiac control. II. Noninvasive indices and basal response as revealed by autonomic blockades. Psychophysiology, 31, 586 - 598.

Carter, H., \& Glick, P. C. (1970). Marriage and Divorce: A Social and Economic Study. Cambridge, MA: Harvard University Press.

Cassell, J. G. (1976). The contribution of the social environment to host resistance. 
American Journal of Epidemiology, 104, 107-123.

Christenfeld, N., Gerin, W., Linden, W., Sanders, M., Mathur, J., Deich, J. D., Pickering, T. G. (1997). Social support effects on cardiovascular reactivity: Is a stranger as effective as a friend? Psychosomatic Medicine, 59, 388-398.

Clarkson, T. B., Manuck, S. B., \& Kaplan, J. R. (1986). Potential role of cardiovascular reactivity in artherogenesis. In K. A. Matthews, S. M. Weiss, T. Detre, T. M. Dembrowski, B. Falkner, S. B. Manuck, \& R. B. Williams (Eds.), Handbook of Stress, Reactivity, and Cardiovascular Disease (pp. 35-47). New York: Wiley.

Cohen, S., Kaplan, J. R., \& Manuck, S. B. (1994). Social support and coronary heart disease: underlying psychological and biological mechanisms. In S. A. Shumaker \& S. M. Czajowski (Eds.), Social Support and Cardiovascular Disease (pp. 195-221). New York: Plenum Press.

Cottington, E. M., Brock, B. M., House, J. S., \& Hawthorne, V. M. (1985). Psychosocial factors and blood pressure in the Michigan statewide blood pressure study. American Journal of Epidemiology, 121, 515-529.

Edens, J. L., Larkin, K. T., \& Abel, J. L. (1992). The effect of social support and physical touch on cardiovascular reactions to mental stress. Journal of Psychosomatic Research, 36, 371-381.

Folkow, B., Hallback, M., Lundgren, Y., Sivertsson, R., \& Weiss, L. (1973). Importance of adaptive changes in vascular design for establishment of primary hypertension, studied in man and in spontaneously hypertensive rats. Circulation Research, 32-33, 2-13.

Gerin, W., Milner, D., Chawla, S., \& Pickering, T. G. (1995). Social support as a moderator of cardiovascular reactivity in women: A test of the direct effects and buffering hypotheses. 
Psychosomatic Medicine, 57, 16-22.

Gerin, W., Pieper, C., Levy, R., Pickering, T. G. (1992). Social support in social interaction: A moderator of cardiovascular reactivity. Psychosomatic Medicine, 54, 324-336.

Girdler, S. S., Turner, J. R., Sherwood, A., \& Light K. C. (1990). Gender differences in blood pressure control during a variety of behavioral stressors. Psychosomatic Medicine, 52, 571-591.

House, J. S., Landis, K. R., \& Umberson, D. (1988). Social Relationships and Health. Science, 241, 540-545.

Hurwitz, B. E., Nelesen, R. A., Saab, P. E., Nagel, J. H., Spitzer, S. B., Gellman, M. D., McCabe, P. M., Philips, D. J., \& Schneiderman, N. (1993). Differential patterns of dynamic cardiovascular regulation as a function of task. Biological Psychology, 36, 75-95.

Janes, C. R. (1990). Migration, changing gender roles, and stress: The Samoan case. Medical Anthropology, 12, 217-248.

Kamarck, T. W., Annunziato, B., \& Amateau, L. M. (1995). Affiliation moderates the effects of social threat on stress-related cardiovascular responses: Boundary conditions for a laboratory model of social support. Psychosomatic Medicine, 57, 183-194.

Kamarck, T. W., Manuck, S. B., \& Jennings, J. R. (1990). Social support reduces cardiovascular reactivity to psychological challenge: A laboratory model. Psychosomatic Medicine, 52, $42-58$.

Kamarck, T. W., Peterman, A. H., \& Raynor, B. A. (1998). The effects of the social environment on stress-related cardiovascular activation: Current findings, prospects, and implications. Unpublished manuscript. 
Kasprowicz, A. L., Manuck, S. B., Malkoff, S. B., \& Krantz, D. S. (1990). Individual differences in behaviorally evoked cardiovascular response: Temporal stability and hemodynamic patterning. Psychophysiology, 27, 605-619.

Keys, A., Taylor, H. L., Blackburn, H., Brozek, J., Anderson, J. T., \& Somonson, E. (1971). Mortality and coronary heart disease among men studied for 23 years. Archives of Internal Medicine, $\underline{128}, 201-214$.

King, M. G., Burrows, G. D., \& Stanley, G. V. (1983). Measurement of stress and arousal: Validation of the stress/arousal adjective checklist. British Journal of Psychology, 74, 473-479.

Kirschbaum, C., Klauer, T., Filipp, S. H., \& Hellhammer, D.H. (1995). Sex-specific effects of social support on cortisol and subjective responses to acute psychological stress. Psychosomatic Medicine, 57, 23-31.

Kleinke, C. L., \& Williams, G. (1994). Effects of interviewer status, touch, and gender on cardiovascular reactivity. Journal of Social Psychology, 134, 247-249.

Krantz, D. S., \& Manuck, S. B. (1984). Acute psychophysiologic reactivity and risk of cardiovascular disease: A review and methodological critique. Psychological Bulletin, 96, 435-464.

Lawler, K. A., Wilcox, Z. C., \& Anderson, S. F. (1995). Gender differences in patterns of dynamic cardiovascular regulation. Psychosomatic Medicine, 57, 357-365.

Lepore, S. J. (1995). Cynicism, social support, and cardiovascular reactivity. Health Psychology, 14, 210-216.

Lepore, S. J., Allen, K. M., \& Evans, G. W. (1993). Social support lowers cardiovascular reactivity to an acute stressor. Psychosomatic Medicine, 55, 518-524. 
Leppin, A., \& Schwarzer, R. (1990). Social support and physical health: An updated metaanalysis. In L. R. Schmidt, P. Schwenkmezger, J. Weinman, \& S. Maes (Eds.) Theoretical and Applied Aspects of Health Psychology (pp. 185-202). New York: Harwood Academic Publishers. MacKay, C., Cox, T., Burrows, G., \& Lazzerini, T. (1978). An inventory for the measurement of self-reported stress and arousal. British Journal of Social and Clinical Psychology, 17, 283-284.

Manuck, S. B., Kaplan, J. R., \& Clarkson, T. B. (1983). Behaviorally induced heart rate reactivity and atherosclerosis in Cynomolgus monkeys. Psychosomatic Medicine, 45, 95-108.

Merck Manual, 14th Edition. (1982). P. 378.

Miller, J. C., \& Horvath, S. M. (1978). Impedance cardiography. Psychophysiology, 15, 8091.

Miller, R. S., \& Lefcourt, H. M. (1982). The assessment of social intimacy. $\underline{\text { Journal of }}$ Personality Assessment, 46, 514-518.

Rosenman, R. H. (1990). Cardiovascular reactivity: Physiological or psychological. In L. R. Schmidt, P. Schwenkmezger, J. Weinman, \& S. Maes (Eds.) Theoretical and Applied Aspects of Health Psychology ( pp. 283-295). New York: Harwood Academic Publishers.

Saab, P. G. (1989) Cardiovascular and neuroendocrine responses to challenge in males and females. In N. Schneiderman, S. M. Weiss, \& P. G. Kaufman (Eds), Handbook of Research Methods in Cardiovascular Behavioral Medicine (pp. 453-481). New York: Plenum Press.

Sarason, I. G., Levine, H. M., Basham, R. B., \&Sarason, B. R. (1983). Assessing social support: the Social Support Questionnaire. Journal of Personality and Social Psychology, 44, 127-139.

Seligman, M. E. (1972). Learned Helplessness, Annual Review of Medicine, 23, 407. 
Selye, H. (1956). The Stress of Life, McGraw-Hill: New York.

Sheffield, D., \& Carroll, D. (1994). Social support and cardiovascular reactions to active laboratory stressors. Psychology and Health, 9, 305-316.

Sherwood, A., Dolan, C. A., \& Light, K. C. (1990). Hemodynamics of blood pressure responses during active and passive coping. Psychophysiology, 27, 656-668.

Sherwood, A., \& Turner, J. R. (1992). A conceptual and methodological overview of cardiovascular reactivity research. In J. R. Turner, A. Sherwood, \& K. C. Light (Eds.), Individual differences in cardiovascular response to stress (pp. 3 - 32). New York: Plenum Press.

Tardy, C. H. (1985). Social support measurement. American Journal of Community Psychology, 13, 187-202.

Thorsteinsson, E. B., James, J. E., \& Gregg, M. E. (1998). Effects of video-relayed social support on hemodynamic reactivity and salivary cortisol during laboratory-based behavioral challenge. Health Psychology, 17, 436-444.

Uchino, B. N., Cacioppo, J. T., \& Kiecolt-Glaser, J. K. (1996). The relationship between social support and physiological processes: A review with emphasis on underlying mechanisms and implications for health. Psychological Bulletin, 119, 488-531.

Uchino, B. N., \& Garvey, T. S. (1997). The availability of social support reduces cardiovascular reactivity to acute psychological stress. Journal of Behavioral Medicine, 20, 15-27. Watson, S. R., \& Friend, R. (1969). Measurement of social-evaluative anxiety. Journal of Consulting and Clinical Psychology, 83, 448-457.

Wilson, M. F., Lovallo, W. R., \& Pincomb, G. A. (1989). Noninvasive measurement of 
cardiac functions. In N. Schneiderman, S. M. Weiss, \& P. G. Kaufman (Eds.), $\underline{\text { Handbook of Research }}$ Methods in Cardiovascular Behavioral Medicine (pp. 23 - 50). New York: Plenum Press. 
Table 1

Descriptive Statistics for Demographic Variables by Gender $(\mathrm{n}=34)$

\begin{tabular}{|c|c|c|c|c|c|c|}
\hline \multirow[t]{2}{*}{ Parameter } & \multicolumn{2}{|c|}{ Men $(n=17)$} & \multicolumn{2}{|c|}{ Females $(\mathrm{n}=17)$} & \multirow[b]{2}{*}{$\underline{F}$} & \multirow[b]{2}{*}{$\underline{p}$} \\
\hline & $\underline{\mathrm{M}}$ & $\underline{\mathrm{SD}}$ & $\underline{\mathrm{M}}$ & $\underline{\mathrm{SD}}$ & & \\
\hline Age (yrs) & 20.59 & $(2.76)$ & 20.88 & $(5.43)$ & .04 & $>.05$ \\
\hline Height (inches) & 69.5 & $(2.00)$ & 64.00 & $(2.46)$ & 51.23 & $<.001$ \\
\hline Weight (lbs) & 168.18 & $(25.35)$ & 127.82 & $(22.71)$ & 23.91 & $<.001$ \\
\hline BSA & 1.92 & $(.13)$ & 1.60 & $(.15)$ & 45.91 & $<.001$ \\
\hline Years of Education & 13.94 & $(1.20)$ & 13.47 & $(.80)$ & 1.82 & $>.05$ \\
\hline \multicolumn{7}{|l|}{ Duration of } \\
\hline Relationship (mths) & 16.88 & (20.81) & 22.53 & $(24.84)$ & .516 & $>.05$ \\
\hline Sleep (hours) & 7.65 & $(1.43)$ & 8.18 & $(1.41)$ & 1.18 & $>.05$ \\
\hline FNE & 10.59 & $(6.97)$ & 14.76 & $(8.41)$ & 2.49 & $>.05$ \\
\hline MSIS & 154.94 & (12.13) & 151.65 & $(28.20)$ & .20 & $>.05$ \\
\hline SSQ6 Before & 33.18 & $(1.78)$ & 31.41 & $(3.91)$ & 2.88 & $>.05$ \\
\hline SSQ6 After & 32.82 & $(2.38)$ & 28.76 & $(5.80)$ & 7.12 & $<.02$ \\
\hline
\end{tabular}

Note. Values enclosed in parentheses represent standard deviations. BSA = body surface area; FNE = Fear of Negative Evaluation; MSIS = Miller Social Intimacy; SSQ = Social Support Questionnaire. 
Table 2

Descriptive Statistics (Standard Deviations in parentheses) for all cardiovascular parameters

chronologically during Rest, Preparation, and Speech phases $(n=34)$

\begin{tabular}{|c|c|c|c|c|c|c|}
\hline Parameter & Rest 1 & Prep 1 & Speech 1 & Rest 2 & Prep 2 & Speech 2 \\
\hline \multirow[t]{2}{*}{ SBP* } & $113.72^{\mathrm{a}}$ & 119.66 & 132.50 & $117.66^{\mathrm{b}}$ & 119.69 & 131.60 \\
\hline & (10.89) & $(9.78)$ & $(12.51)$ & (11.91) & (13.21) & $(14.83)$ \\
\hline \multirow[t]{2}{*}{ DBP } & 73.00 & 79.15 & 86.99 & 74.39 & 78.34 & 85.37 \\
\hline & $(8.95)$ & $(10.81)$ & (14.01) & (11.34) & $(10.44)$ & (14.68) \\
\hline \multirow[t]{2}{*}{$\mathrm{HR}^{*}$} & $72.92^{\mathrm{a}}$ & 80.32 & $87.97^{\mathrm{c}}$ & $75.58^{b}$ & 80.04 & $83.82^{\mathrm{d}}$ \\
\hline & (11.06) & (11.52) & (17.88) & (10.18) & (12.89) & $(14.46)$ \\
\hline \multirow[t]{2}{*}{ PEP* } & $103.67^{\mathrm{a}}$ & 98.42 & $92.26^{\mathrm{c}}$ & $100.41^{\mathrm{b}}$ & 98.81 & $96.30^{\mathrm{d}}$ \\
\hline & (12.79) & (14.36) & (18.07) & $(12.26)$ & $(12.80)$ & (15.73) \\
\hline \multirow[t]{2}{*}{ SI* } & $58.22^{\mathrm{a}}$ & 53.08 & 52.61 & $56.22^{b}$ & 52.00 & 51.16 \\
\hline & $(15.50)$ & $(14.82)$ & (13.81) & (14.92) & (13.36) & $(12.71)$ \\
\hline \multirow[t]{2}{*}{ CI } & 4.24 & 4.24 & 4.51 & 4.22 & 4.18 & 4.33 \\
\hline & (1.09) & $(1.14)$ & $(1.30)$ & (1.09) & (1.04) & (1.37) \\
\hline \multirow[t]{2}{*}{ TPR } & 992.21 & 1896.08 & 1972.15 & 1017.82 & 1884.32 & 2077.66 \\
\hline & $(256.63)$ & $(622.42)$ & (655.69) & (252.49) & $(542.64)$ & $(813.27)$ \\
\hline
\end{tabular}


Note. $* \mathrm{p}<.05$; means with superscripts of $\mathrm{a}$ and $\mathrm{b}$ indicate significant differences in resting measures; means with superscripts of $\mathrm{c}$ and d indicate significant differences in measures during the speech task. $\mathrm{SBP}=$ systolic blood pressure, $\mathrm{DBP}=$ diastolic blood pressure, $\mathrm{HR}=$ heart rate, $\mathrm{PEP}=$ pre-ejection period, $\mathrm{SI}=$ stroke index, $\mathrm{CI}=$ cardiac index, $\mathrm{TPR}=$ total peripheral resistance. 
Table 3

Descriptive statistics for Stress and Arousal Subscales of the Stress/Arousal Checklist (chronologically and by Condition)

After Speech \#1

Stress Score

Arousal Score
$27.91(7.08)$

$23.94(6.01)$
After Speech \#2

$30.32(6.83)$

$23.62(6.26)$ 
Table 4

$\underline{\text { Partial correlation coefficients for MSIS and FNE scores with cardiovascular difference by Condition }}$

\begin{tabular}{|c|c|c|c|}
\hline Parameter & Modified SSQ Score & MSIS Score & FNE Score \\
\hline & $\mathrm{r} \quad(\mathrm{p})$ & $\mathrm{r} \quad(\underline{\mathrm{p}})$ & $\mathrm{r} \quad(\mathrm{p})$ \\
\hline SBP Alone & $-.21(.24)$ & $.23(.19)$ & $-.04(.81)$ \\
\hline SBP With Partner & $-.20(.27)$ & $-.02(.93)$ & $.14(.44)$ \\
\hline DBP Alone & $-.20(.27)$ & $.28(.11)$ & $-.02(.91)$ \\
\hline DBP With Partner & $.06(.72)$ & $-.08(.64)$ & $.09(.64)$ \\
\hline HR Alone & $-.17(.34)$ & $-.13(.48)$ & $.04(.82)$ \\
\hline HR With Partner & $-.20(.27)$ & $-.12(.48)$ & $.10(.58)$ \\
\hline PEP Alone & $.21(.24)$ & $.32(.07)$ & $-.09(.61)$ \\
\hline PEP With Partner & $.12(.50)$ & $.13(.48)$ & $.11(.54)$ \\
\hline SI Alone & $-.10(.58)$ & $-.13(.48)$ & $.00(.98)$ \\
\hline SI With Partner & $-.06(.72)$ & $.08(.68)$ & $.03(.86)$ \\
\hline CI Alone & $-.16(.37)$ & $-.32(.07)$ & $.14(.43)$ \\
\hline CI With Partner & $-.13(.46)$ & $-.21(.25)$ & $.19(.28)$ \\
\hline TPR Alone & $.15(.41)$ & $.17(.33)$ & $-.15(.42)$ \\
\hline TPR With Partner & $.13(.48)$ & $.02(.92)$ & $.01(.98)$ \\
\hline
\end{tabular}


Note. Values enclosed in parentheses represent standard deviations. FNE $=$ Fear of Negative Evaluation; MSIS = Miller Social Intimacy Scale; SSQ = Social Support Questionnaire, SBP = systolic blood pressure, $\mathrm{DBP}=$ diastolic blood pressure, $\mathrm{HR}=$ heart rate, $\mathrm{PEP}=$ pre-ejection period, $\mathrm{SI}=$ stroke index, $\mathrm{CI}=$ cardiac index, $\mathrm{TPR}=$ total peripheral resistance. 


\section{Figure Captions}

Figure 1. DBP (mm Hg) by Order (Partner Present First, No Partner Present First) and Period (i.e., first speech, second speech).

Figure 2. Adjusted Mean HR (bpm) by Gender and Phase (preparation, speech).

Figure 3. Adjusted Mean PEP (ms) by Gender and Phase (preparation, speech).

Figure 4. Cardiac Index (\% change) by Gender and Phase (preparation, speech).

Figure 5. TPR (\% change) by Gender and Phase (preparation, speech). 


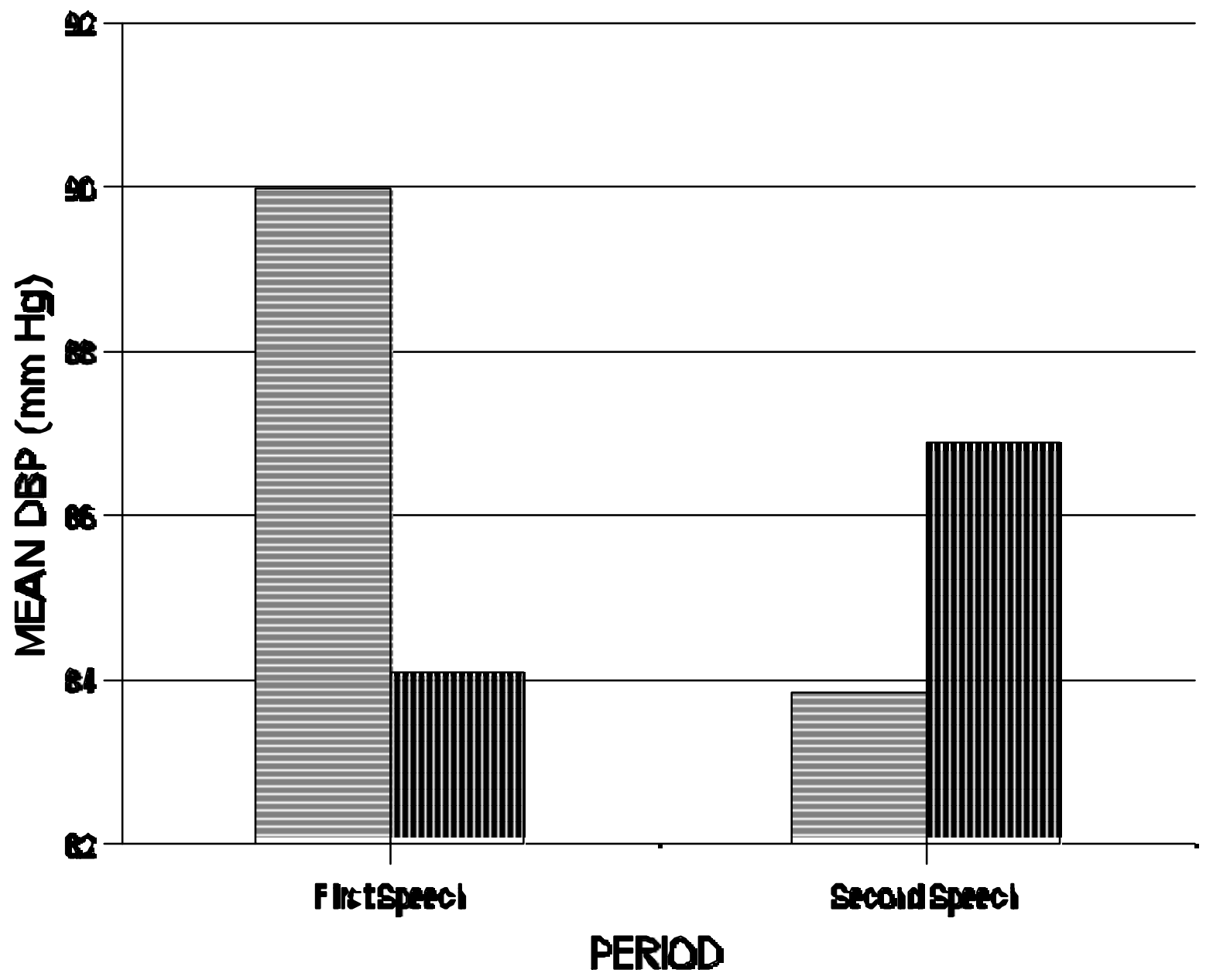

\section{目 PARTNER PRESENT |III ND PARTNER PRESENT}




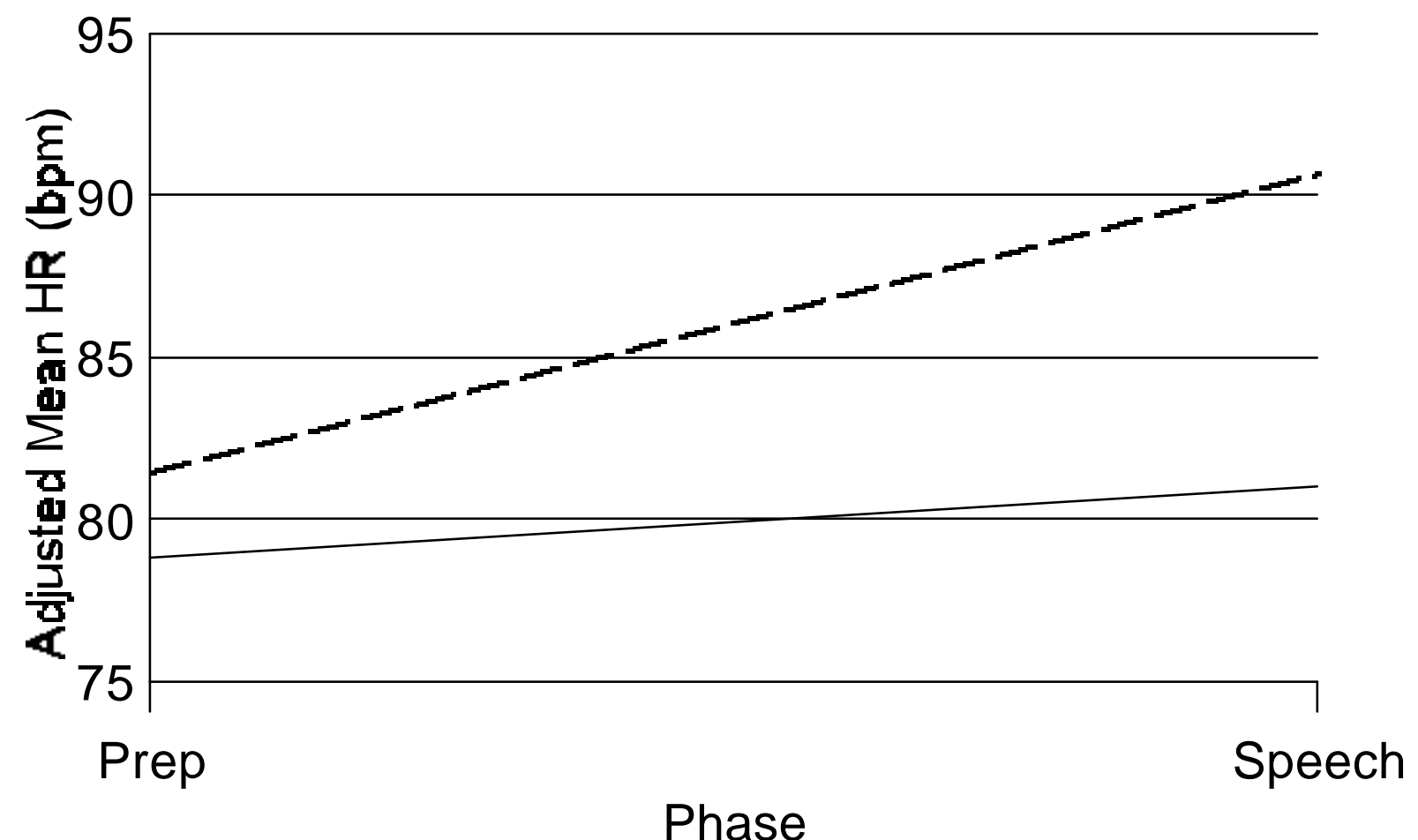

$$
\begin{array}{ll}
--- & \text { Female } \\
& \text { Male }
\end{array}
$$




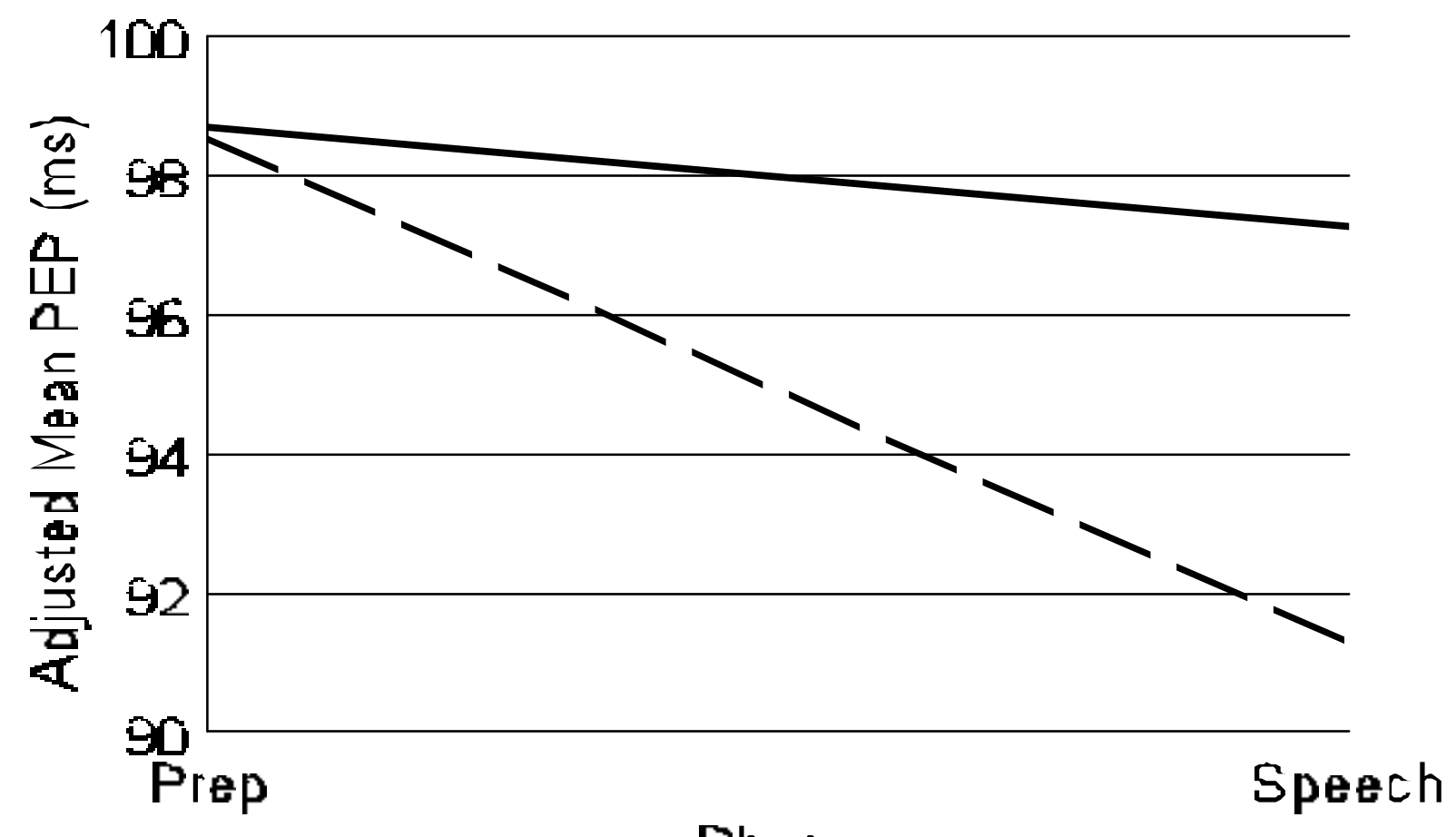

Phase

$$
\begin{aligned}
& - \text { Female } \\
& \text { Male }
\end{aligned}
$$




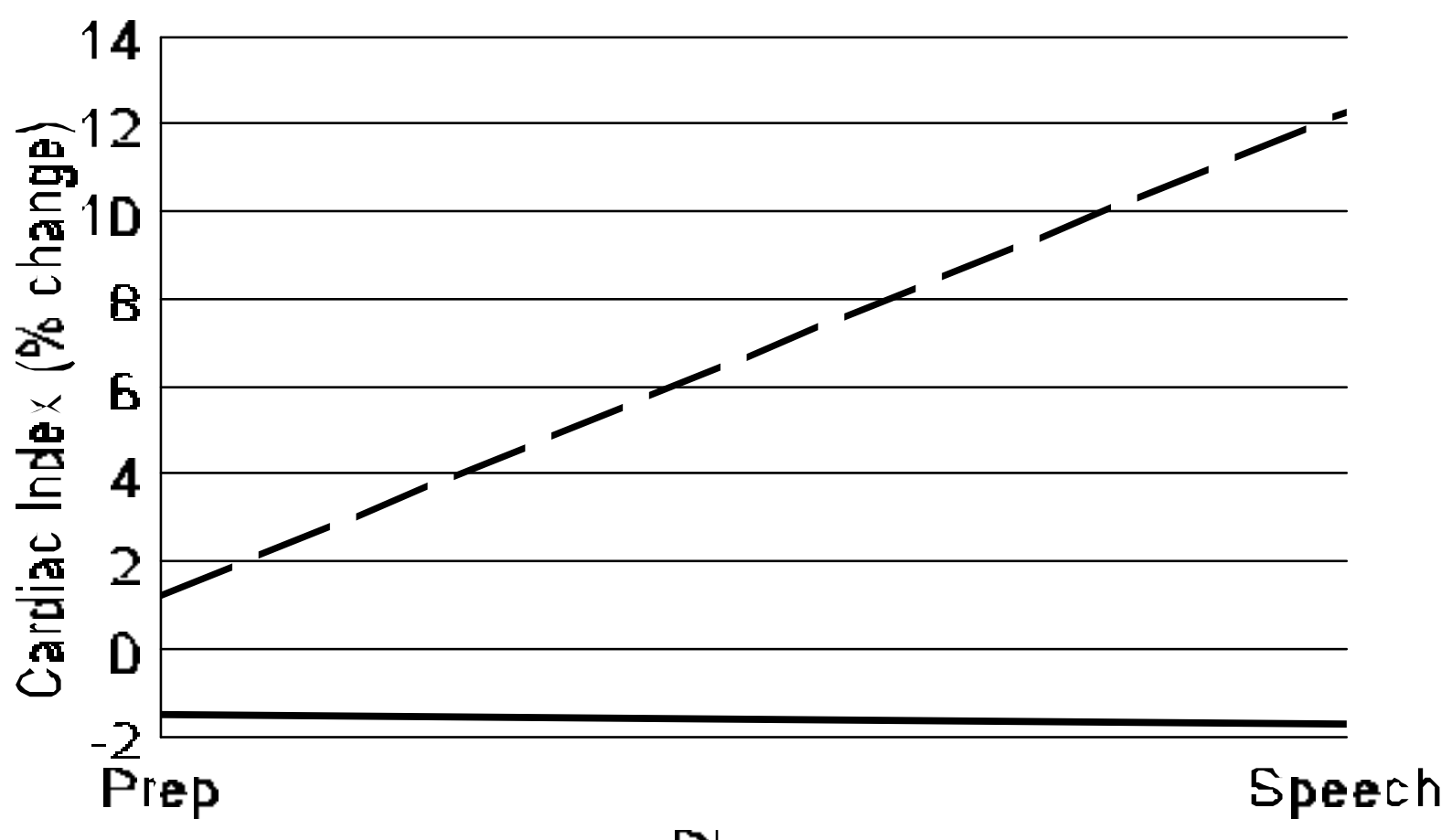

Phase

$$
\longrightarrow \text { Females }
$$




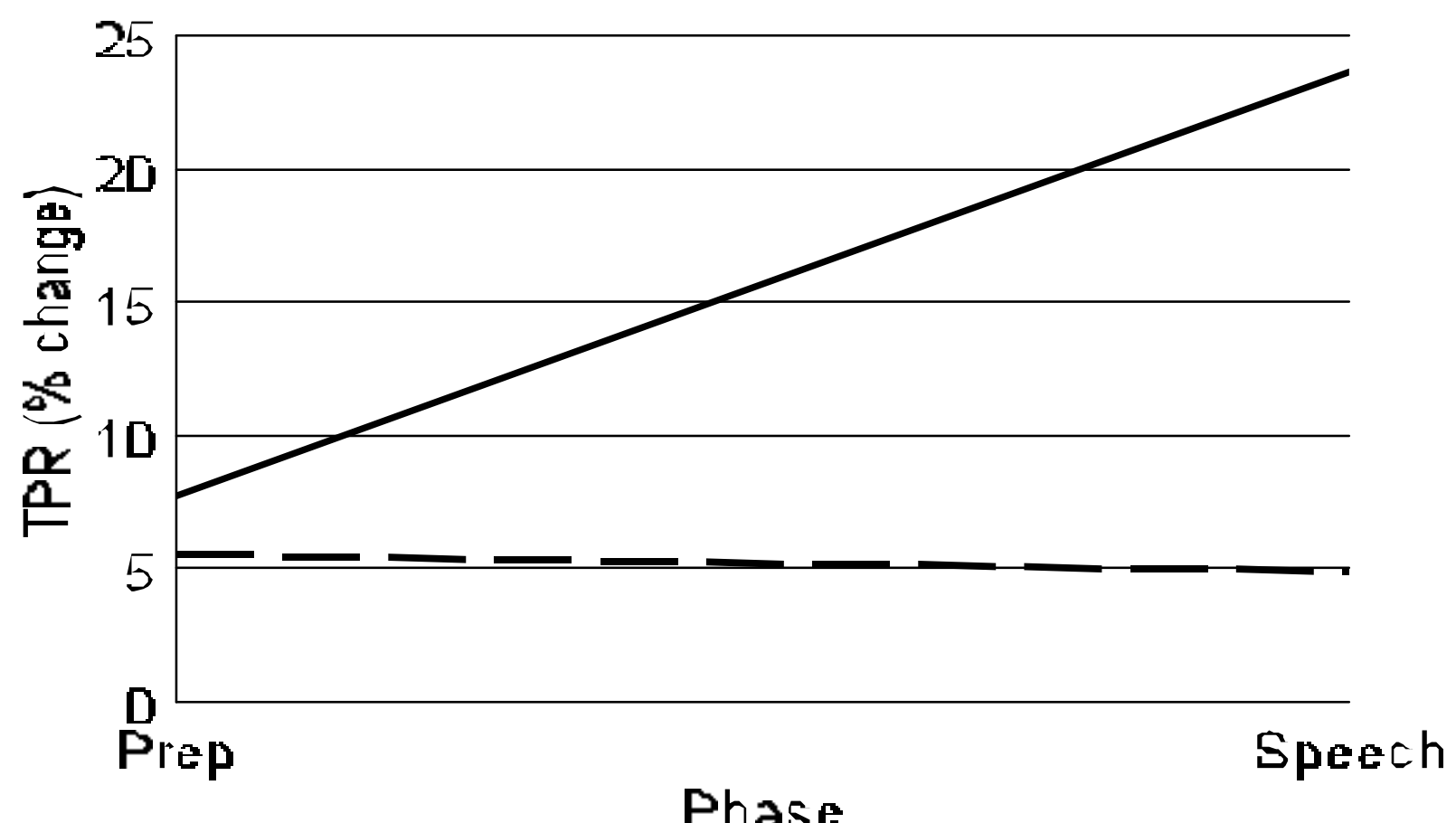

Phase

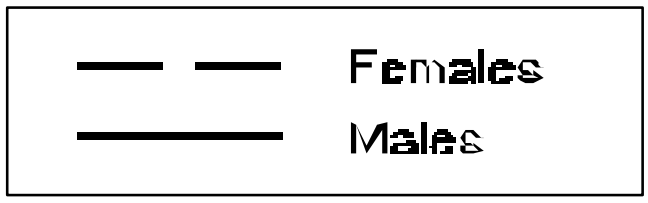




\section{APPENDIX A \\ PARTICIPANT SCREENING PROTOCOL}

1. Do you have any chronic health problem such as diabetes, hypertension, asthma, epilepsy, etc.?

2. Are you on any medications (including oral contraceptives)? Please list medication and associated medical condition.

3. Has a physician ever been told that you have high blood pressure?

4. Do you currently smoke or use smokeless tobacco?

5. How long have you been emotionally involved with your significant other?

5. For women: Date of first day of last menstrual period. 
APPENDIX B

\section{DEMOGRAPHIC SURVEY}

Subject Number

Age

Ethnicity

Weight

Height

Highest Grade Completed

Student? Y N

Date of session

Hours of Sleep last night

Time since Last meal

Time since Last Caffeine

Time since Last Nicotine

Time since Last Alcohol

Time since Last Exercise

Current Medications

Family History of hypertension

Family History? Yes No

If yes, in whom?

Women: Date of first day of last menstrual period 


\section{APPENDIX C \\ PRESCREENING FOR SPEECH TOPIC}

1. I believe in euthanasia if the person involved is very unhappy or in pain.

$\begin{array}{lllccl}\text { Always } & \text { Sometimes } & \text { Neither true } & \text { Sometimes } & \text { Always } & \text { No } \\ \text { True } & \text { True } & \text { nor false } & \text { False } & \text { False } & \text { Opinion }\end{array}$

2. I believe in the death penalty in capital crime cases.

$\begin{array}{lllccl}\text { Always } & \text { Sometimes } & \text { Neither true } & \text { Sometimes } & \text { Always } & \text { No } \\ \text { True } & \text { True } & \text { nor false } & \text { False } & \text { False } & \text { Opinion }\end{array}$

3. I believe in a woman's right to an abortion if she wants it.

$\begin{array}{lllccl}\text { Always } & \text { Sometimes } & \text { Neither true } & \text { Sometimes } & \text { Always } & \text { No } \\ \text { True } & \text { True } & \text { nor false } & \text { False } & \text { False } & \text { Opinion }\end{array}$




\section{APPENDIX D \\ SOCIAL SUPPORT QUESTIONNAIRE (MODIFIED)}

Instructions: Please answer the following questions with regards to the support you feel that your partner provided during the speech task.

1) How satisfied were you with the overall support your partner provided to distract you from your worries if you felt under stress?

$\begin{array}{llllll}1 & 2 & 3 & 4 & 5 & 6 \\ \text { Very } & & & & \text { Very } \\ \text { Dissatisfied } & & & & & \text { Satisfied }\end{array}$

2) How satisfied were you with your partner's ability to help you feel more relaxed if you felt under pressure or tense?

$\begin{array}{llllll}1 & 2 & 3 & 4 & 5 & 6 \\ \text { Very } & & & & & \text { Very } \\ & & & & \end{array}$

Dissatisfied $\quad$ Satisfied

3) How satisfied were you with the degree to which your partner accepted you totally, including both your worst and your best points?

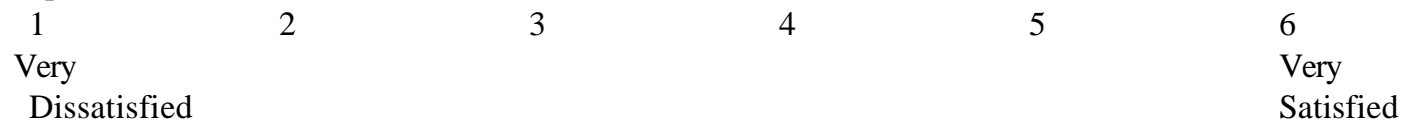

4) How satisfied were you with your ability to count on your partner to care about you, regardless of what was happening to you?
1
2
3
4
5
6
Dissatisfied
Very
Satisfied

5) How satisfied were you with your partner's ability to help you feel better if you were feeling generally down-in-thedumps?

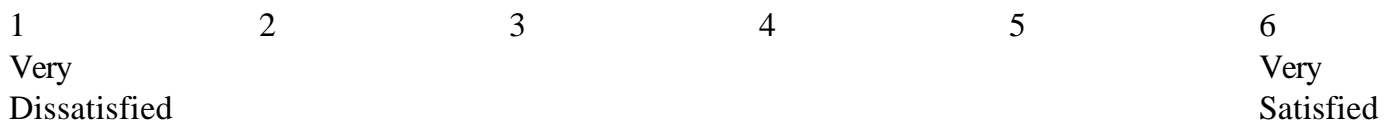

6) How satisfied were you with your partner's ability to console you if you were very upset?

$\begin{array}{llllll}1 & 2 & 3 & 4 & 5 & 6 \\ \text { Very } & & & & \text { Very } \\ \text { Dissatisfied } & & & & \text { Satisfied }\end{array}$




\section{CURRICULUM VITAE}

Lynda M. Ciano-Federoff

March 1999

\section{Education}

West Virginia University, Morgantown, WV

Program: Doctoral candidate in APA-accredited Ph.D. program in Adult Clinical Psychology

(Expected date of graduation: August 1999)

Dissertation: "Effects of gender and social support on cardiovascular reactivity to a speech task" (Chairperson: Kevin Larkin, Ph.D.)

San Jose State University, San Jose, California

Degrees: Master of Arts in Psychology, 1994; Bachelor of Arts in Psychology, with Honors, 1992

Master's Thesis: "Measuring codependency in breast cancer survivors" (Chairperson: Gregory Feist, Ph.D.).

Honors Thesis: "How attitudes may affect the manifestation of breast cancer" (Chairperson: Nusha Askari. Ph.D.).

\section{Professional Experience: Clinical}

Intern, Penn State Geisinger Medical Center, Danville, PA (APA accredited Clinical Psychology Internship; Director: Paul Kettlewell, Ph.D., ABPP, June 1998 - present).

Graduate Assistant, Ruby Memorial Hospital Pain Management Center, Morgantown, WV (August 1997 - June 1998). Supervisor: Jeannie Sperry Clark, Ph.D.

Intern Therapist, Valley Comprehensive Community Mental Health Center, Morgantown, WV (August 1996 - October 1997). Supervisors: Justin Semack, MS, William Fremouw, Ph.D., and Kevin Larkin, Ph.D.

Student Therapist, West Virginia University, Quin Curtis Center, Morgantown, WV (August 1995 June 1998). Supervisors: Jan Kouzes, Ph.D. and Kevin Larkin, Ph.D.

Volunteer Activities, $\quad$ 1. American Cancer Society, Monterey, CA (June 1989-May 1995)

2. American Cancer Society, Santa Cruz, CA (June 1988 - May 1995).

3. Visiting Nurse Association, Hospice Group, Gilroy, CA. (August 1990- May 1995). 